\title{
A Novel Online Detection System for Wheelset Size in Railway Transportation
}

\author{
Xiaoqing Cheng, ${ }^{1,2}$ Yuejian Chen, ${ }^{1,3}$ Zongyi Xing, ${ }^{4}$ Yifan $\mathrm{Li}^{3,5}$ and Yong Qin \\ ${ }^{1}$ State Key Laboratory of Rail Traffic Control and Safety, Beijing Jiaotong University, Beijing 100044, China \\ ${ }^{2}$ School of Traffic and Transportation, Beijing Jiaotong University, Beijing 100044, China \\ ${ }^{3}$ Department of Mechanical Engineering, University of Alberta, Edmonton, AB, Canada T6G 2R3 \\ ${ }^{4}$ School of Automation, Nanjing University of Science and Technology, Nanjing, Jiangsu 210094, China \\ ${ }^{5}$ School of Mechanical Engineering, Southwest Jiaotong University, Chengdu, Sichuan 610031, China
}

Correspondence should be addressed to Yuejian Chen; yuejian1@ualberta.ca

Received 10 February 2016; Revised 9 March 2016; Accepted 5 April 2016

Academic Editor: Rafael Morales

Copyright (c) 2016 Xiaoqing Cheng et al. This is an open access article distributed under the Creative Commons Attribution License, which permits unrestricted use, distribution, and reproduction in any medium, provided the original work is properly cited.

The online detection of wheelset size has important implications for ensuring the safety of railway operation and decreasing the maintenance costs. Based on laser displacement sensors (LDS), a novel online detection system of the wheel size is proposed using only six two-dimensional LDS and two one-dimensional LDS. The calculation principles of tread profile and wheel diameter are given, as well as the calibration method. Errors induced by wheel-rail vibration, misalignment, sensor noise, S-shape running, and wheelset differential are also analyzed. After system implementation, field experiments were performed using both standard wheel and several real trains. It turns out that the detection uncertainty of flange width and height is $0.1 \mathrm{~mm}$ and wheel diameter $0.3 \mathrm{~mm}$, which can meet the requirements of maintenance.

\section{Introduction}

Wheel and rail interact with each other by designed profiles and geometric parameters. The wear of the profile significantly influences the dynamic performance of railway vehicles and even leads to derailment in a massive stage [1]. Therefore, at the very beginning of railway transportation, measuring and ensuring of wheel-rail interactions are a fundamental issue [2]. With the continuous increasing of axle load, train speed, and higher reliability requirement, this issue attracts more attention than ever.

According to the charge-coupled device (UIC) 510-2 code, the geometric parameters of wheelset that need to be measured consist of diameter and tread profile managed by flange width and flange height [3]. Varieties of measuring techniques have appeared, such as specially designed calipers, hand-on automatic scanner, and online detection system [4].

At the earlier stage, the caliper is an effective tool for measuring wheelset size because of the advantage of the simple operation. However, it has shortages of high labor intensity and fluctuated accuracy depends on the skillfulness of workers. Meanwhile, calipers, a contact measuring tool, will inevitably undermine the measuring wheel, causing certain damage.

After that, advanced artificial caliper has emerged with applying noncontact technology. One recognized tool is MiniProf Wheel System developed by Greenwood Engineering [5]. The MiniProf Wheel System is magnetically attached to the wheel. It provides the calculation of wear parameters and is also capable of measuring the flange and taper line diameter on wheels. Due to the benefits of utilizing computer analysis, overall efficiency is increased but this system still takes more than five minutes to measure a single car, not to mention the whole train. Medianu et al. [6] also developed a hand-on scanning system for tread profile. This system uses one-dimensional LDS (1D-LDS) driven by worn gear to scan tread profile. On the whole, those hand-on systems require the train to remain static or even dismantled, facing a great challenge of detection efficiency. 
A widely distinguished technology is online detection system which has the advantages of noncontact, high efficiency, and high accuracy. The high efficiency is realized by dynamic measurement; namely, a train passes the measurement system at a certain speed. There are some commercial companies, such as MERMEC Group [7], IEM, Inc. [8], and KLD Labs, Inc. [9], selling wheelset detection systems on the market. These systems mainly utilize structured laser light and charge-coupled device (CCD) image processing technology. Chen et al. [10] and Gong et al. [11] proposed a structured laser light and CCD based online detection system for tread profile and diameter, respectively. In their research, two pairs of structured laser light and CCD are adopted to recover the inner and outer profiles of each wheel and register them by the iterative closest point algorithm. In diameter detection, the cycloid constraint is utilized to obtain a wide distribution of the contact points. Even though many possible factors that cause the error are considered, the system still lacks real data validation and statistical detection uncertainty analysis. Mian et al. [12] provided an optical evaluation method for railway wheelset with installing image cameras along at least one circumference of the wheel. Such a system can be of high price with so many optical sensors. Gao et al. [13] utilized a pair of line structured laser light and CCD to obtain multiple contact points via repeated shooting. This method needed to measure the speed of the train precisely, and the space interval of the points was decided by the speed and the time interval of shooting. Zhang et al. [14] used only one CCD camera to capture the image of the light profile of the wheelset, and, in the meantime, the tread profile is illuminated by a linear laser. Overall, the structure of structured laser light and CCD based system seems to be too complex. Such a structure also brings about difficulty in the calibration of those systems. The combination of structures of structured laser light and CCD is also sensitive to the harsh environment with vibration and light.

Apart from the structured laser light and CCD sensors, LDS can provide more satisfactory results. The LDS is a special kind of structured light-vision measurement sensor where the photoelectrical detector and laser light source are assembled, providing benefits like easy installation and no need to calibrate intrinsic parameters online. Russian scientists [15] reported their innovative laser sensor, claiming that 1D-LDS can be enough for measuring tread profile. Then, they [16] further derived a mathematical calculation regarding wheel diameter detection. However, the method needs a high precise train speed and time interval of shooting. Gao et al. [17] utilized one 1D-LDS and two eddy current sensors to detect the wheel diameter. Wu and Chen [18] used high-speed CCD and 1D-LDS to measure the diameter and the accuracy was within $1.2 \mathrm{~mm}$. Zhang et al. [19] used two 1D-LDS and a position sensor to detect the wheel diameter and, meanwhile, wavelet analysis is used to eliminate the signal noise. Triangle geometry was the main computational algorithm in the LDS method. These systems do not need a precise train speed and time interval of shooting anymore. However, the 1D-LDS methods mentioned above needed the dot laser to be strictly projected at the contact point on the

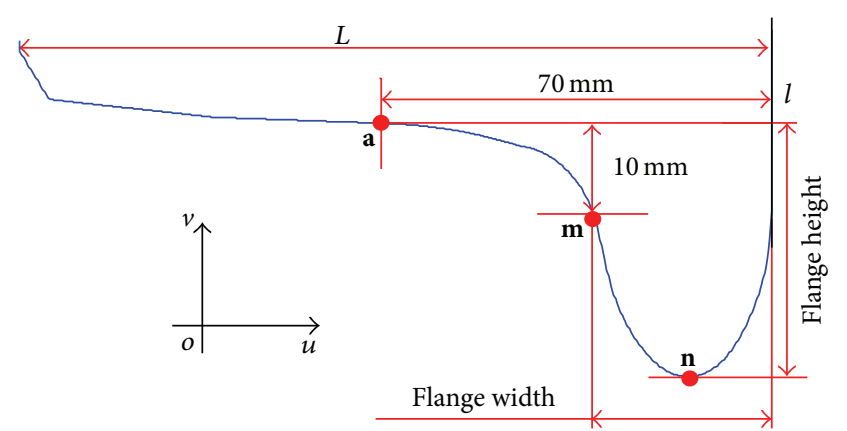

FIGURE 1: Schematic of measuring target.

wheel tread. That is difficult to achieve because of the S-shape motion of the wheelset.

Aiming at LDS based detection system, the authors previously proposed an online detection system using eight 2D-LDS to detect the wheel diameter and tread profile [20]. The system utilized a digital I/O card to generate digital synchronous signals which guarantee simultaneous working of all sensors. The 2D-LDS sensors are relatively of a high price and in the previous system some of the 2D-LDS are actually not frequently used. In this paper, the authors replaced several 2D-LDS sensors with 1D-LDS. Six 2D-LDS and two 1D-LDS are implemented in this new system. Working simultaneously, the data collected from all the sensors are processed, and then wheel diameter and tread profile are calculated. Errors induced by wheel-rail vibration, sensor noise, misalignment, S-shape running, and wheelset diameter differential are also analyzed. At last, after the system is implemented, the field test is carried out by standard wheelset test and real train test.

\section{System Principle}

2.1. Measuring Target. Figure 1 shows a typical wear tread profile [21]. We define the coordinate uov as the tread profile panel across the wheel center. The inner side of the wheel, as shown in black line $l$ in Figure 1, has no wear-out and deformation when there is wheel-rail contact. $L$ is the wheel hub thickness, namely, the distance from the inner side to the outer side of the wheel. The base point $\mathbf{a}$, which is the center of the vertical wheel load and is considered to be the diameter point of the wheel, is $-70 \mathrm{~mm}$ away from the line $l$ along $u$ axis. The base point $\mathbf{m}$, which is the center of the lateral wheel load, is $-10 \mathrm{~mm}$ away from base point a along $v$-axis. The base point $\mathbf{n}$ is the vertex point of the wheel rim. The tread profile is somehow complex so that the condition of tread profile is usually evaluated by flange width and height. The flange width is defined by the width between base line $l$ and base point $\mathbf{m}$ along $u$-axis and the flange height is defined by the distance between base point $\mathbf{a}$ and vertex point $\mathbf{n}$ along $v$-axis.

2.2. System Layout. The presented wheelset size online detection system depends on LDS sensors. Figure 2 shows the system layout. The system consists of six 2D-LDS and two 1DLDS, each of which is installed below the track to measure 




FIGURE 2: Schematic diagram of sensors installation.

both sides of the tread profile and diameter. They can be divided into two groups because the left side and right side are mirrored. Taking the left side LDS as an example, the 2DLDS L1 and L3, together with 1D-LDS L2, measure the wheel diameter by three points' principle. The 2D-LDS L3 and L4 measure the tread profile.

Both the 2D and 1D laser sensors are based on laser triangulation measurement principle and are made up of laser diode and a CCD linear sensor element. The emitted laser forms a laser belt on the wheel tread and then the laser is reflected to the CCD linear inductive components. Inside the sensor, there is an integrated circuit unit to process the optical displacement data and to obtain the tread and flange profile coordinates. Based on the principle that the output points of the LDS are originated from the laser emitting source, in application, the laser emitting source should be regarded as the origin of the scanning coordinate. The signal from all the sensors is transmitted to IPC through data acquisition port. A digital $\mathrm{I} / \mathrm{O}$ card is utilized to produce precisely a $1 \mathrm{kHz}$ square signal in order to ensure all sensors to complete the task of acquiring the tread profile synchronously. The sensors begin to collect the data on the decline of the square wave signal and then transmit the data to the IPC through data acquisition port for the subsequent process. The sampled signal is analyzed in data processing software, and, finally, the condition of each wheel is decided. There is also an optical fiber in the IPC so that the condition of each wheel can be transmitted to distant depot office. All the sensors are fixed by special designed mechanical sensor fixture so that the sensors can be installed in certain space position. The fixtures are supported by the well manufactured mechanical structure. The whole system is finally connected with the ground by the pedestal.

In addition, the system also consists of several accessorial types of equipment that have not been shown in system layout figure, which are wheel position sensor and automatic train identification antenna. Three wheel position sensors are installed beside the outside of the rail. Along the rail, the first one is used to detect the arriving moment of the first wheel axis of a train and, hence, to trigger subsequent hardware facilities; the second one is used to trigger the scanning of all laser sensors; the last one is used to detect the leaving moment of the last wheel axis of a train and, hence, to close the subsequent hardware facilities.

2.3. Static Tread Profile Calculation Principle. Taking the left side LDS as an example, we define world coordinate frame (WCF) $o-x y z$ and LDS scanning coordinates $o^{(1)}-x^{(1)} y^{(1)} z^{(1)}$, $o^{(2)}-x^{(2)} y^{(2)} z^{(2)}, o^{(3)}-x^{(3)} y^{(3)} z^{(3)}$, and $o^{(4)}-x^{(4)} y^{(4)} z^{(4)}$ for L1, 


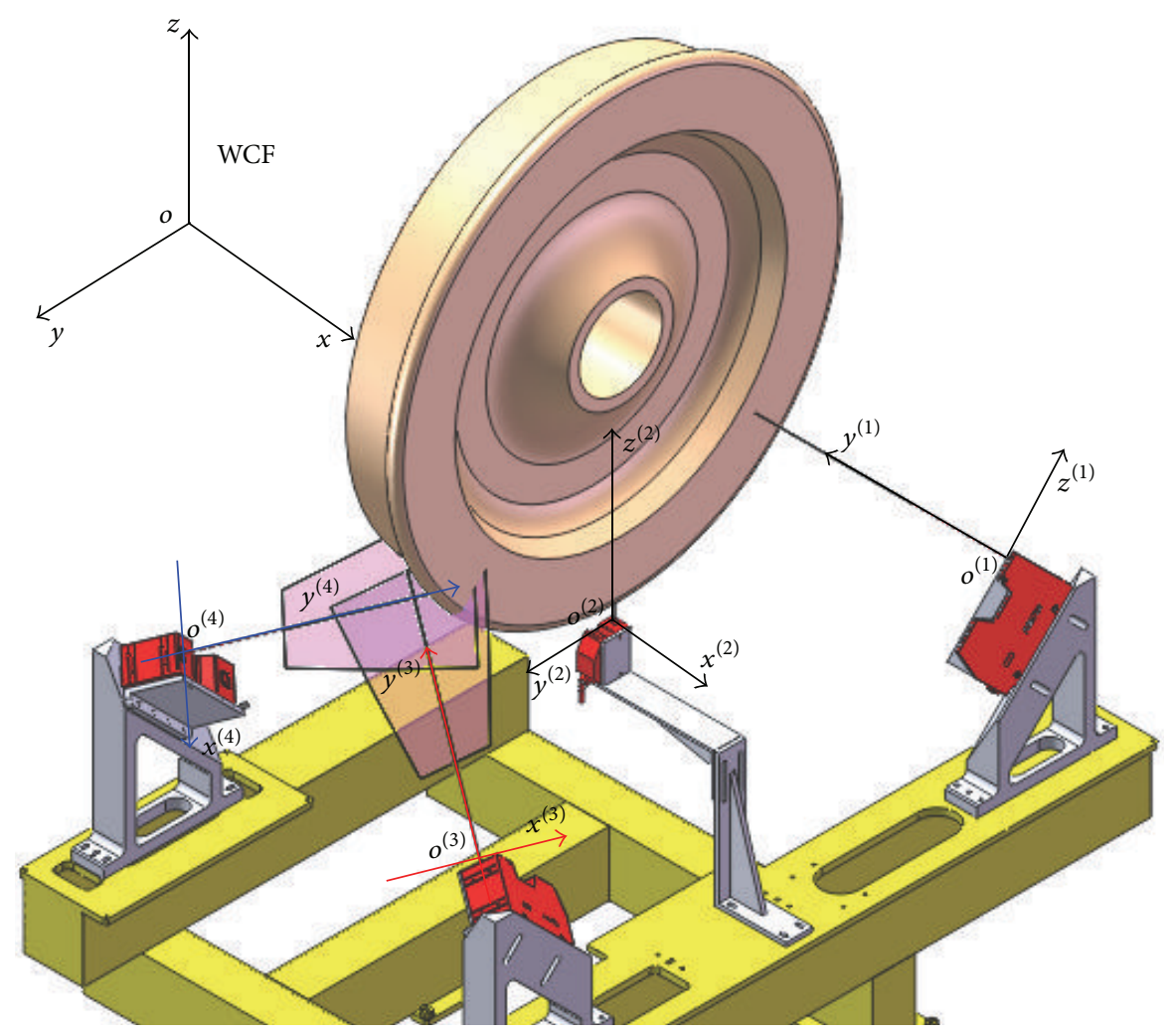

FIGURE 3: Coordinates set.

L2, L3, and L4, respectively. As shown in Figure 3, the scanning coordinates take the origin of laser light as the coordinate origin, the equal angle bisector of the triangle laser panel as $y$-axis, and the direction in triangle laser panel orthogonal with $y$-axis as $x$-axis and finally use right-hand rule to determine $z$-axis. The coordinates of the output points are in the scanning coordinate system of the sensor.

The wheelset is assumed to be in the right position and to remain static. The scanning coordinate of L3 and L4 is rotated by two angles from the WCF, namely, angles $\alpha$ and $\beta$ with respect to $x$-axis and $y$-axis. The tread profile is measured by L3 and L4 and the scanning panels of L3 and L4 are the same. The tread profile can be measured in tread profile panel constituted by L3 and L4. So, as shown in Figure 4(a), only angle $\beta$ is considered when measuring tread profile. Because of angle $\beta$, the output line is distorted and needs to be transformed into physical profile. According to the installation angles, $\beta_{3}$ and $\beta_{4}$, the output data is transformed by

$$
\begin{aligned}
u_{n}^{(3)} & =\sqrt{x_{n}^{(3)^{2}}+y_{n}^{(3)^{2}}} \sin \left(\theta+\beta_{3}\right) \\
& =x_{n}^{(3)} \cos \beta_{3}+y_{n}^{(3)} \sin \beta_{3}, \\
v_{n}^{(3)} & =\sqrt{x_{n}^{(3)^{2}}+y_{n}^{(3)^{2}}} \cos \left(\theta+\beta_{3}\right) \\
& =y_{n}^{(3)} \cos \beta_{3}-x_{n}^{(3)} \sin \beta_{3},
\end{aligned}
$$

$$
\begin{aligned}
u_{n}^{(4)} & =\sqrt{x_{n}^{(4)^{2}}+y_{n}^{(4)^{2}}} \sin \left(\theta^{\prime}-\beta_{4}\right) \\
& =x_{n}^{(4)} \cos \beta_{2}-y_{n}^{(4)} \sin \beta_{2}, \\
v_{n}^{(4)} & =\sqrt{x_{n}^{(4)^{2}}+y_{n}^{(4)^{2}}} \cos \left(\theta^{\prime}-\beta_{4}\right) \\
& =y_{n}^{(4)} \cos \beta_{2}+x_{n}^{(4)} \sin \beta_{2},
\end{aligned}
$$

where $\left(x_{n}^{(3)}, y_{n}^{(3)}\right)$ and $\left(x_{n}^{(4)}, y_{n}^{(4)}\right)$ are detected dot in LDS scanning coordinates $o^{(3)}-x^{(3)} y^{(3)} z^{(3)}$ and $o^{(4)}-x^{(4)} y^{(4)} z^{(4)} ; \theta$ is the angle between $y^{(3)}$-axis and the line that connects origin $o^{(3)}$ and detected dot; $\theta^{\prime}$ is the angle between $y^{(4)}$-axis and the line that connects origin $o^{(4)}$ and detected dot; $\left(u_{n}^{(3)}, v_{n}^{(3)}\right)$ is the coordinate value of detected dot in the new coordinate $u^{(3)} o^{(3)} v^{(3)}$ and $\left(u_{n}^{(4)}, v_{n}^{(4)}\right)$ is the coordinate value of detected dot in the new coordinate $u^{(4)} o^{(4)} v^{(4)}$ as well.

After transformation, the scanned lines in two different coordinates, $u^{(3)} o^{(3)} v^{(3)}$ and $u^{(4)} o^{(4)} v^{(4)}$, need to be merged into one coordinate. We define the coordinate $u^{(3)} o^{(3)} v^{(3)}$ as tread profile base coordinate uov and move all the data from $u^{(4)} o^{(4)} v^{(4)}$ into uov by (3), as shown in Figure 4(b). Hence,

$$
\begin{aligned}
& u_{n}=u_{n}^{(4)}+\Delta u \\
& v_{n}=v_{n}^{(4)}+\Delta v
\end{aligned}
$$




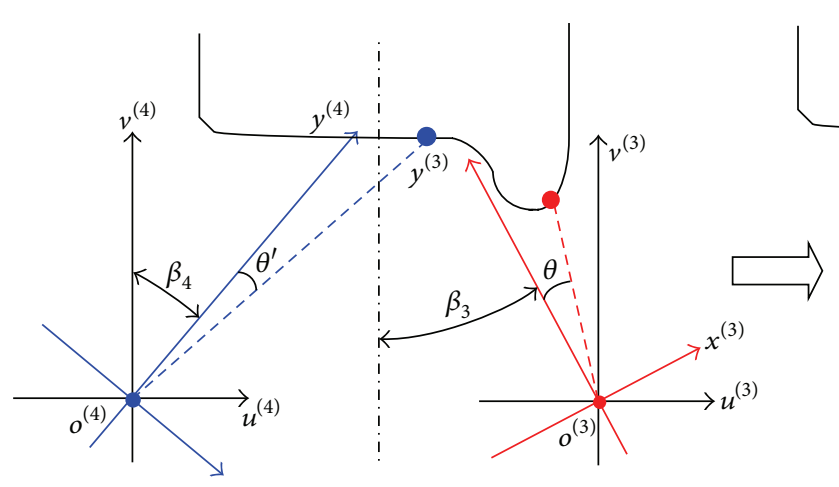

(a)

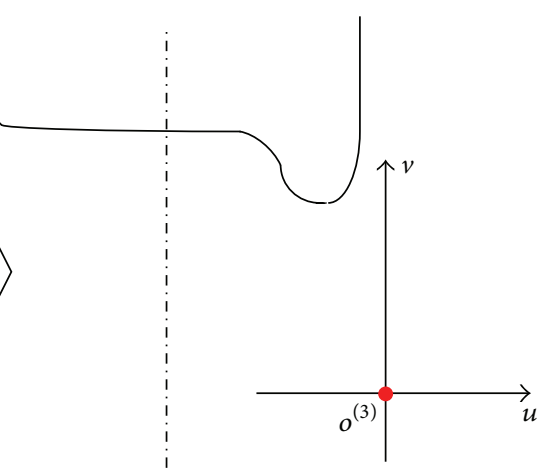

(b)

FIgURE 4: (a) Coordinate transformation of L4 and coordinate transformation of L3. (b) Moving all the data from $u^{(4)} o^{(4)} v^{(4)}$ to $u o v$.

where $\left(u_{n}, v_{n}\right)$ is the dot in tread profile base coordinate $u o v$; $\Delta u$ and $\Delta v$ are the offset from $u^{(4)} o^{(4)} v^{(4)}$ to $u o v$.

As we know, flange width, flange height, and wheel diameter are determined by several base points and base line. The output points from sensors are discrete, so base points $\mathbf{a}$, $\mathbf{m}$, and $\mathbf{n}$ are more likely not in one of the scanned points. The output points are also polluted with sensor noise, which induced more detection uncertainty when we directly regard it as the base points. Over here, curve fitting is used for extracting the base point, as well as the base line. Through this method, the coordinate value of base points can be precisely extracted and the sensor noise can also be eliminated to some extent. It is difficult to use a single curve to fit all the tread due to the complexity of tread contour. Therefore, fitting discrete points of each base point within a certain range is applied to improve the accuracy of the extracted base point coordinate value. The common method of curve fitting is the least square method [22]. The least square method uses a given set of measured data to get the functional relation $f\left(x, a_{0}, a_{1}, \ldots, a_{n}\right)$ between the variable $x$ and the variable $y$ based on the principle of least squares. Then, the weighted sum of squares' value of the residual $e_{k}$ between the fitting function and the actual measured value at each point can be minimal, which means $F$ in (4) is minimal:

$$
F=\sum_{i=0}^{I} \omega\left(x_{i}\right)\left(f_{i}-y_{i}\right)^{2},
$$

where $\omega\left(x_{i}\right) \geq 0$ is the weight which reflects the notion that the data $\left(x_{i}, y_{i}\right)$ accounts for the proportion in the experiment; $I$ denotes the number of data points. According to the tread profile features and experimental research, fourth-order polynomial $y=\sum_{i=0}^{4} a_{i} x^{4-i}$ is selected to fit each subsection curve based on the least square method.

With curve fitting technique, four lines in total are fitted in order to extract the coordinate value of base points $\mathbf{a}, \mathbf{m}$, and $\mathbf{n}$. As shown in Figure 5, at first, the inner side of the wheel has no wear-out and deformation when there is wheel-rail contact, so base line $l$ is fitted by selecting all the data points in the inner side of the wheel. The base point $\mathbf{a}$ is $70 \mathrm{~mm}$ away from the base line $l$ along $u$-axis. Then, the green line is fitted in order to extract base point a by selecting data points within

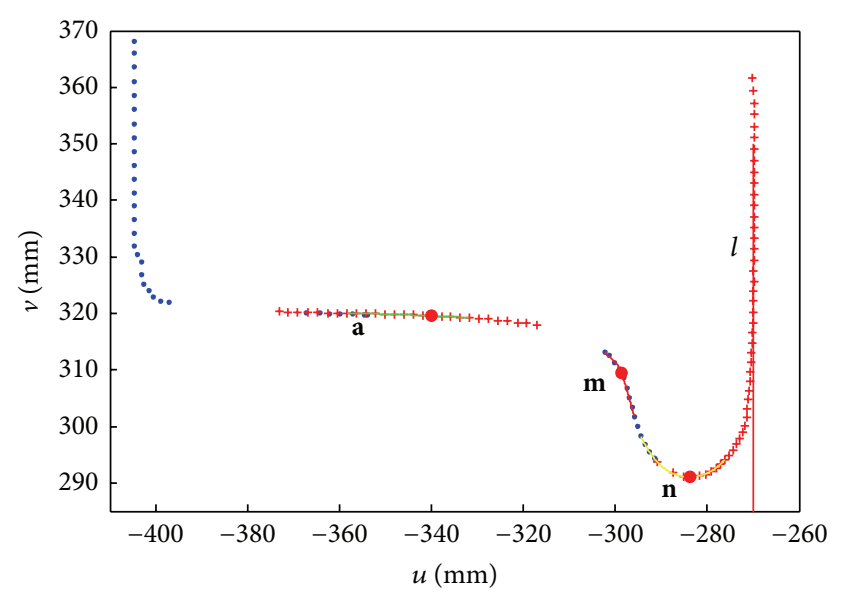

FIgURE 5: Curve fitting results.

a certain range of base point $\mathbf{a}$. The red line and yellow line are also fitted by the same method in order to extract base points $\mathbf{m}$ and $\mathbf{n}$, respectively.

After four lines are obtained, the precise coordinate value of all base points can be determined. To this end, the flange height and flange width are calculated as follows:

$$
\begin{gathered}
F_{w}=u_{l}-u_{m}, \\
F_{h}=v_{n}-v_{a},
\end{gathered}
$$

where $F_{w}$ is flange width; $F_{h}$ is flange height; $u_{l}$ is the $u$-axis coordinate value of base line $l ; u_{m}$ is the $u$-axis coordinate value of base point $\mathbf{m} ; v_{n}$ is the $v$-axis coordinate value of base point $\mathbf{n} ; v_{a}$ is the $v$-axis coordinate value of base point $\mathbf{a}$.

\subsection{Static Wheel Diameter Calculation Principle. Wheel} diameter is detected by 2D-L1, 1D-L2, and 2D-L3. Each one of the LDS measures one point in the circular wheel so that the wheel diameter can be determined by three points.

The wheelset is assumed to be in the right position and to remain static. The coordinates of the output points are in the scanning coordinate system of the sensor. Similar to tread profile calculation, the coordinate transformation 


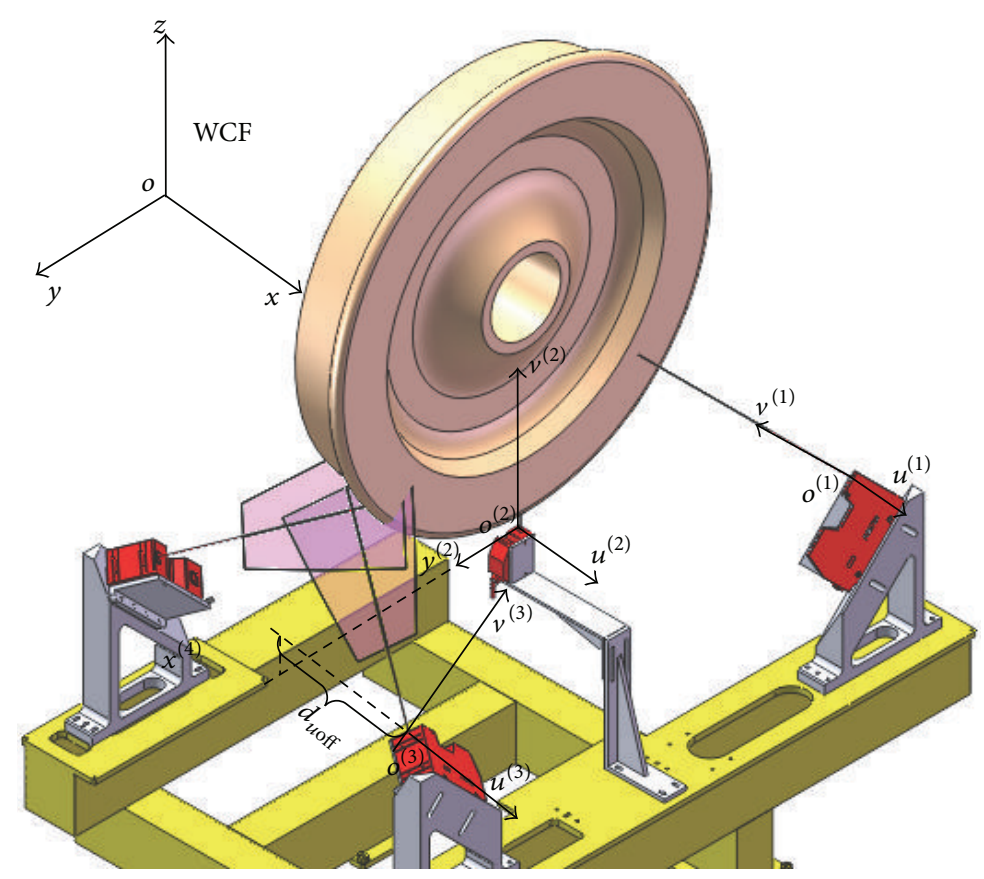

Figure 6: Scanning coordinates of 2D-L1, 1D-L2, and 2D-L3 after coordinate transformation.

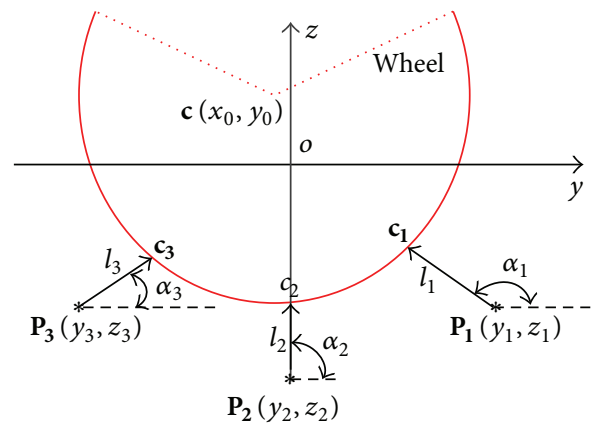

(a)

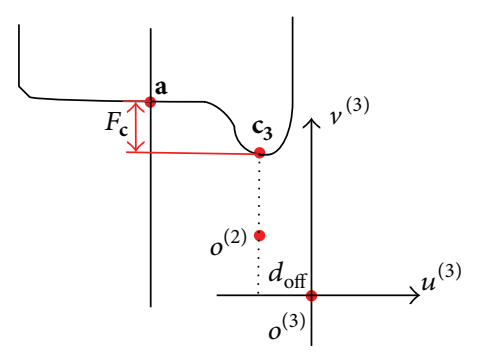

(b)

FIGURE 7: Wheel diameter calculation principle in two dimensions: (a) sight along the $x$-axis; scanning coordinates of 2D-L1, 1D-L2, and 2D-L3 after transformation; (b) sight in the $u^{(3)} o^{(3)} v^{(3)}$ coordinate.

was conducted and the scanning coordinates $u^{(1)} o^{(1)} v^{(1)}$, $u^{(2)} o^{(2)} v^{(2)}$, and $u^{(3)} o^{(3)} v^{(3)}$ for 2D-L1, 1D-L2, and 2D-L3, respectively, have been obtained. Figure 6 shows the scanning coordinates of 2D-L1, 1D-L2, and 2D-L3 after coordinate transformation. Notice that $u^{(2)} o^{(2)} v^{(2)}$ is still the same as $y^{(2)} o^{(2)} z^{(2)}$ because of the installation position of 1D-L2. Figure 6 also shows the offset $d_{\text {off }}$ between the origin of the coordinate $u^{(3)} o^{(3)} v^{(3)}$ and laser scanning line of L2 in $u^{(2)}$ axis. Among three points, the two points detected by $2 \mathrm{D}-\mathrm{L} 1$ and $2 \mathrm{D}-\mathrm{L} 3$ are extracted from the $2 \mathrm{D}$ profiles. The offset $d_{\text {off }}$ is the $u^{(2)}$-axis coordinate value to extract the points in the flange circle from two-dimensional profile. This offset $d_{\text {off }}$ is determined by sensor installation.

Figure 7 shows the wheel diameter calculation principle in two dimensions where (a) shows the principle that three points determine a diameter in yoz WCF and (b) shows extracting the point in the flange circle among twodimensional profile and the final wheel diameter distance subtraction by $F_{c}$. From Figure $7(a)$, the installation of each LDS is modeled as three parameters in yoz WCF, which are the position $\mathbf{P}_{\mathbf{i}}\left(y_{i}, z_{i}\right)$ and angle $\alpha_{i}$. They determine the position of laser origin and the direction of detection, respectively. The angle $\alpha_{2}$ for $1 \mathrm{D}-\mathrm{L} 2$ is designed as $\pi / 2$. The positions $\mathbf{P}_{\mathbf{1}}$ and $\mathbf{P}_{\mathbf{3}}$ are designed as symmetric with respect to the scanning line of $1 \mathrm{D}-\mathrm{L} 2$, as well as the angles $\alpha_{1}$ and $\alpha_{3}$. Even though many of the parameters are designed to be equal, for instance, $z_{1}=z_{3}$, the real parameters will vary after engineering implementation due to errors such as installation error and manufacturing error. Thus, this nine-parameter model is proposed for diameter calculation 
because it can describe all the possible errors. The real installation parameters are obtained through calibration, as later described. Moreover, the distances $l_{1}, l_{2}$, and $l_{3}$ are detected from three LDS sensors. The three points $\mathbf{c}_{1}, \mathbf{c}_{2}$, and $\mathbf{c}_{3}$ are in the flange of wheel detected by three sensors, respectively. The point $\mathbf{c}\left(y_{0}, z_{0}\right)$ is the origin of the detected wheel which is calculated by three points $c_{1}, c_{2}$, and $c_{3}$.

According to Figures 6 and 7(a), the first information we can get from the LDS sensors is the laser scanned distances $l_{1}, l_{2}$, and $l_{3} . l_{2}$ is directly detected by $1 \mathrm{D}-\mathrm{L} 2 . l_{1}$ and $l_{3}$ are extracted from the $2 \mathrm{D}$ profiles detected by $2 \mathrm{D}-\mathrm{L} 1$ and $2 \mathrm{D}$ L3, respectively. To extract $l_{1}$ and $l_{3}$, we need to find the correct points in the two-dimensional tread profile. As shown in Figures 6 and $7(\mathrm{~b})$, the point that determines $l_{1}$ and $l_{3}$ should be in the $u$-axis value of offset $d_{\text {off }}$. Similar to detecting tread profile, we use the same curve fitting method to obtain a curve in the contour of the wheel in the uov coordinate that is denoted by $v=f_{3}(u)$. When the curve line is obtained, $l_{3}=v_{3}=f_{3}\left(d_{\text {off }}\right)$, namely, the $v$-axis value of curve $f_{3}(u)$ when $u=d_{\text {off }}$. Similarly, $l_{1}$ is detected by 2D-LDS L1 using the same method as deciding $l_{3}$.

Once the laser scanned distances $l_{1}, l_{2}$, and $l_{3}$ are determined, we get the three points $\mathbf{c}_{1}, \mathbf{c}_{2}$, and $\mathbf{c}_{3}$ in WCF coordinate yoz by

$$
\begin{aligned}
& y_{c_{1}}=y_{1}+l_{1} \sin \alpha_{1}, \\
& z_{c_{1}}=z_{1}+l_{1} \cos \alpha_{1}, \\
& y_{c_{2}}=y_{2}+l_{2} \sin \alpha_{2}, \\
& z_{c_{2}}=z_{2}+l_{2} \cos \alpha_{2}, \\
& y_{c_{3}}=y_{3}+l_{3} \sin \alpha_{3}, \\
& z_{c_{3}}=z_{3}+l_{3} \cos \alpha_{3} .
\end{aligned}
$$

Based on three points $\mathbf{c}_{\mathbf{1}}, \mathbf{c}_{\mathbf{2}}$, and $\mathbf{c}_{\mathbf{3}}$, the wheel center $\mathbf{c}\left(y_{0}, z_{0}\right)$ is determined by

$$
\begin{aligned}
& y_{0}=\frac{\left(z_{c_{1}}-z_{c_{3}}\right)\left(y_{c_{1}}^{2}-y_{c_{2}}^{2}+z_{c_{1}}^{2}-z_{c_{2}}^{2}\right)-\left(z_{c_{1}}-z_{c_{2}}\right)\left(y_{c_{1}}^{2}-y_{c_{3}}^{2}+z_{c_{1}}^{2}-z_{c_{3}}^{2}\right)}{2\left(y_{c_{1}}-y_{c_{2}}\right)\left(z_{c_{1}}-z_{c_{3}}\right)-2\left(y_{c_{1}}-y_{c_{3}}\right)\left(z_{c_{1}}-z_{c_{2}}\right)}, \\
& z_{0}=\frac{\left(y_{c_{1}}-y_{c_{2}}\right)\left(y_{c_{1}}^{2}-y_{c_{3}}^{2}+z_{c_{1}}^{2}-z_{c_{3}}^{2}\right)-\left(y_{c_{1}}-y_{c_{3}}\right)\left(y_{c_{1}}^{2}-y_{c_{2}}^{2}+z_{c_{1}}^{2}-z_{c_{2}}^{2}\right)}{2\left(y_{c_{1}}-y_{c_{2}}\right)\left(z_{c_{1}}-z_{c_{3}}\right)-2\left(y_{c_{1}}-y_{c_{3}}\right)\left(z_{c_{1}}-z_{c_{2}}\right)}
\end{aligned}
$$

and the wheel diameter $D_{r}$ is determined by

$$
D_{r}=2 \cdot \sqrt{\left(y_{0}\right)^{2}+\left(z_{0}-z_{c_{2}}\right)^{2}} \text {. }
$$

From Figure $7(\mathrm{~b}), F_{c}$ is the distance between points $\mathbf{c}_{\mathbf{3}}$ and a along $v$-axis. The wheel diameter detected by the previous three points is somewhere in the contour circle governed by the 1D-L2 only. The point $\mathbf{a}$ is considered to be the diameter point of the wheel which is $-70 \mathrm{~mm}$ away from the inner side of the wheel. In order to obtain the final wheel diameter, we need to further subtract the distance $F_{c}$ from the wheel diameter:

$$
D=D_{r}-2 F_{c}
$$

where $F_{c}$ is the distance between point $c_{\mathbf{3}}$ and point a in $v$-axis (as shown in Figure 7(b)); namely, $F_{c}=v_{a}-v_{c_{3}}$.

2.5. Dynamics Detection. The calculation principles shown above are in static case. When the train passes dynamically, multiscans can be obtained and the misalignment phenomenon caused from wheel passing will occur.

For tread profile detection, ideally, the laser light panel of L3 and L4 is assumed to include the center of the measured wheel. In dynamics detection, it is impossible to meet that assumption for all measured wheels due to the moving of wheel and the discrete sampling of LDS signal. Basically, if the laser light panel does not include the center of the measured wheel, the detected profile is horizontally stretched along $v$-axis. That will lead to the increase of detected flange height and flange width. This phenomenon is called the misalignment between the laser panel and the detection target [9].

Figure 8 shows the dynamics positions of the wheel center and laser panel constituted by L3 and L4 in two-dimensional WCF. The wheel moves forward with a constant speed of $v$. $\mathrm{O}_{1}, \mathrm{O}_{2}$, and $\mathrm{O}_{i}$ are the center of wheel diameter circle under different positions. The laser panel has an installation angle $\alpha_{3}$ with respect to $y$-axis, which can be determined as $z=$ $\tan \alpha_{3} \cdot y$ in WCF. The center points of wheel diameter circle are calculated by (7). $d_{i}$ denote the distance between $i$ th wheel center point and the laser panel.

Theoretically, for every $i$ th position of the wheel, the distance $d_{i}$ from the wheel center $\mathbf{c}\left(y_{0_{i}}, z_{0_{i}}\right)$ to the laser panel can be determined by point to the distance formula as follows:

$$
d_{i}=\left|\frac{\tan \left(\alpha_{3}\right) y_{0_{i}}-z_{0_{i}}}{\sqrt{\tan ^{2}\left(\alpha_{3}\right)+1}}\right| .
$$

When the distance $d_{i}$ equals zero, the wheel center $\mathbf{c}\left(y_{0_{i}}, z_{0_{i}}\right)$ is in the laser panel where the flange height and flange width have no stretching. On the other hand, the bigger the distance $d_{i}$ is, the farther away the wheel center $\mathrm{c}\left(y_{0_{i}}, z_{0_{i}}\right)$ is from the laser panel.

It is worth mentioning that the LDS works when the angle between laser light and detected surface is within a certain range and the angle is influenced by laser wavelength, surface 


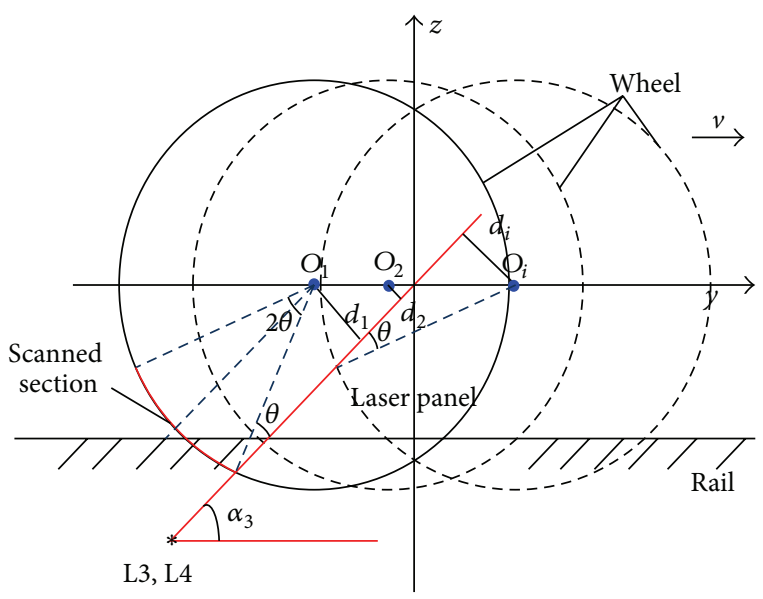

FIgURE 8: Dynamics position of the wheel center.

smoothness, surface material, and so forth [23]. It is assumed that the angle $\theta$ (as shown in Figure 8) is the largest angle at which the LDS can still receive effective scan. When the wheel is moving out of the detection range, the LDS will be unable to scan. Thus, the scanned section will be the arc with a central angle of $2 \theta$, and all the tread profiles and diameters are scanned from this section. For most LDS sensors, the angle $\theta$ can reach up to $45^{\circ}$, so the system can measure $90^{\circ}$ arc of the wheel. Correspondingly, the maximum value of the distance $d_{i}$ is $R \sin \theta$, where $R$ is the wheel radius.

The misalignment phenomenon will bring about certain error to the profile detection. Among all the effective scans, we must select those scans where the induced error is acceptable. In this paper, the error induced in the tread profile detection is analyzed in Section 3. As a result, the error is directly proportional to the distance $d_{i}$. So, we set up a certain threshold $K p$. When the distance $d_{i}<K p$, the detected tread profile can be regarded as useful profiles where the error induced by the misalignment phenomenon is negligible. The threshold $K p$ is firstly obtained through error analysis and also is adjustable according to the field experiment. Due to the benefits from the high sampling frequency of the LDS sensors, $M$ times of scans can be obtained for a wheelset. Then, we can remove the bulky error first and perform average operation to get the final wheel flange and wheel width as follows:

$$
\begin{aligned}
& F_{w_{f}}=\frac{1}{M} \sum_{i=1}^{M} F_{w_{i}}, \\
& F_{h_{f}}=\frac{1}{M} \sum_{i=1}^{M} F_{h_{i}},
\end{aligned}
$$

where $F_{w_{i}}$ and $F_{h_{i}}$ are the flange width and flange height in $i$ th scan, respectively; $F_{w_{f}}$ and $F_{h_{f}}$ are the final flange width and flange height, respectively. The average operation here can reduce the final error caused by Gaussian sensor noise.

For wheel diameter detection, the three points that determine the wheel diameter are always in the contour circle. Thus, the calculation results will not be influenced by different wheel positions. However, $F_{c_{i}}$ in every $i$ th scan will still be stretched and bring about some error. Similarly, we select a set of scans by comparing whether the distance $d_{i}$ is smaller than a certain threshold $K d$ or not. When $d_{i}<$ $K d$, the error induced in the detected $F_{c}$ is negligible. The two thresholds, $K d$ and $K p$, might be different because of the different detection error requirements for tread profile and wheel diameter. In this way, $N$ times of scans can be obtained. Then, we can remove the bulky error first and perform average algorithm to get the final wheel diameter as follows:

$$
D_{f}=\frac{1}{N} \sum_{i=1}^{N} D_{i},
$$

where $D_{i}$ is the wheel diameter in $i$ th scan; $D_{f}$ is the final wheel diameter.

2.6. Calibration. The measuring and calculating of tread profile and wheel diameter depend on many installation parameters. Regarding tread profile calculation, they are the angle $\beta_{3}$ in (1), angle $\beta_{4}$ in (2), and the offset $\Delta u$ and offset $\Delta v$ in (3). For wheel diameter, they are the offset $d_{\text {off }}$ between the origin of the coordinate uov and laser scanning line of L2 in $u^{(2)}$-axis, the angles $\alpha_{1}, \alpha_{2}$, and $\alpha_{3}$, and the positions $\mathbf{P}_{1}$, $\mathbf{P}_{2}$, and $\mathbf{P}_{3}$ in (6). When the LDS are installed and fixed, it is impossible for those parameters to be the same with designed values because of the manufacture error of mechanical parts and installation accuracy. So, calibration is certainly needed.

During the calibration process for tread profile detection, a standard wheel is placed on the rail over the detection system, and then the offset and rotation angle of the coordinate transformation matrix can be determined. In terms of the angles $\beta_{3}$ and $\beta_{4}$, the calibrated accurate value is to make sure the inner and outer panels of the wheel are vertical. For the offset $\Delta u$, the calibrated accurate value is to make sure the detected wheel hub thickness equals the standard wheel hub thickness and the offset $\Delta v$ is to make sure the scanned profiles from two LDS coincide with each other.

As for the calibration process for wheel diameter, a set of new ground wheelsets is used. The ground wheelset is with different diameters that are $770 \mathrm{~mm}, 790 \mathrm{~mm}, 810 \mathrm{~mm}$, and $840 \mathrm{~mm}$. We set the minimization function $f(x)$ as the squared summation of detected diameters subtracted by real diameter. That is,

$$
\min f(x)=\sum_{i=1}^{J}\left|D_{i}-D_{r}\right|^{2},
$$

where $D$ is the detected diameter according to wheel diameter calibration principle; $D_{r}$ is the real diameters; $x=$ $\left[d_{\text {off }}, \alpha_{1}, \alpha_{1}, \alpha_{3}, y_{1}, z_{1}, y_{2}, z_{2}, y_{3}, z_{3}\right]$ is the variables to be calibrated; $J$ denotes the number of ground wheelsets. MATLAB has provided such tools to solve those optimization problems. Over here, we use fmincon function to minimize the function and the constraints in terms of the variables are also given according to real physical ranges. Finally, the optimal values of the parameters can be obtained. These values are assumed to be the real values that the implemented system has and have been further put into use in system service. 


\section{Detection Error Analysis}

In this section, we consider four factors which are rail vibration, sensor noise, misalignment, and wheel inclination caused by wheel S-shape running and the differential of diameters.

3.1. Wheel-Rail Vibration. Wheel-rail vibration is the first factor that we considered. In our system, all the sensors are well fixed by the mechanical support and mechanical pedestal that has no direct contact with rail. So, the wheel-rail vibration will not directly transmit to the sensors and, instead, the wheel-rail vibration has to transmit to the ground of the depot and then transmit to the sensors through mechanical support and mechanical pedestal. The vibration of the ground is on a lower level, the maximum of acceleration is only $0.4 \mathrm{~m}^{2} / \mathrm{s}$ [24] in Guangzhou metro depot, and it is also attenuated by the mechanical pedestal. We also measured the maximum of acceleration of mechanical support during train passing, which is only $0.2 \mathrm{~m}^{2} / \mathrm{s}$. So, the change of the position of the sensors due to the wheel-rail vibration in our system can be neglected. Furthermore, all the laser sensors are capturing data simultaneously and the exposure time of the LDS is within 50 microseconds. The vibration of the wheel will not cause considerable movement within such a short time. Overall, the system is assumed to be reliable against wheel-rail vibration.

3.2. Misalignment. As previously mentioned in Section 2, Dynamics Detection, if the laser light panel does not include the center of the measured wheel, the detected profile is horizontally stretched along $v$-axis. This phenomenon is called the misalignment between the laser panel and the detection target, which will lead to the increase of the detected flange height and flange width. Chen et al. [10] derived a geometric model regarding how many errors will be generated for flange height when wheel position varies. The error $e$ of the flange height is

$$
e=\sqrt{R_{C}^{2}-d^{2}}-\sqrt{R^{2}-d^{2}}-R_{C}+R,
$$

where $R$ is the wheel radius; $R_{C}$ is the radius in the wheel rim; $d$ is the distance from the wheel center to the laser panel, as described in Section 2.

On the basis of this geometric model, when we know how much the error of the flange height is, the errors of the flange width can be derived accordingly. For different wear wheels, the profiles, as well as the fitted line for lateral contact point $\mathbf{m}$, are certainly different. To illustrate the massiveness of errors, here we chose the same wheel where the fitted line for lateral contact point $\mathbf{m}$ is $v=f(u)$. We obtain the inverse function $u=g(v)$ and stretch it horizontally by a factor of $\left(F_{h}+e\right) / F_{h}$. So, the stretched curve line is

$$
u=g_{2}(v)=g\left(\frac{v F_{h}}{\left(F_{h}+e\right)}\right) .
$$

Eventually, the error of flange width is $\eta=g_{2}(10)-F_{w}$, where $F_{w}$ is the original flange width.

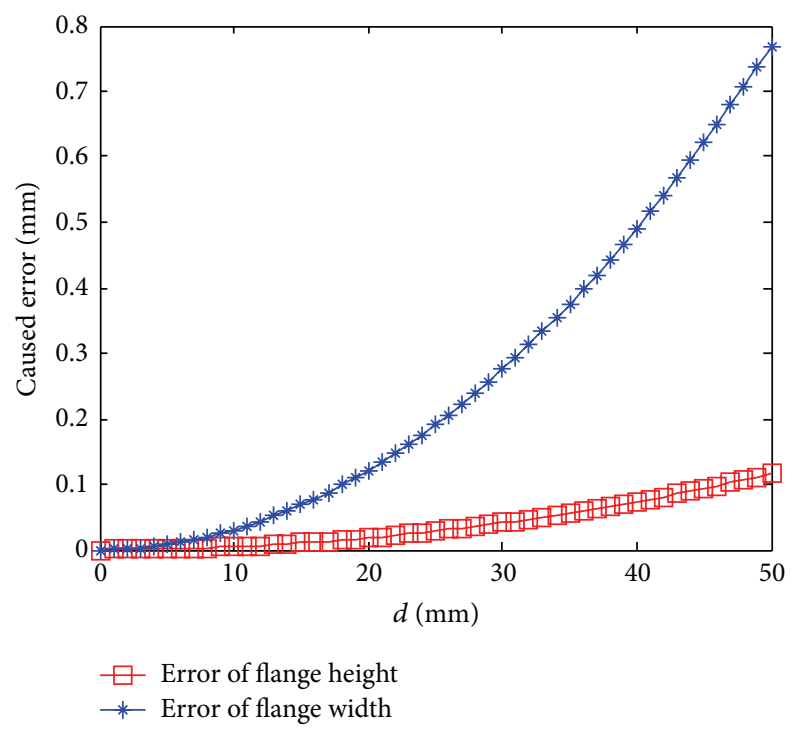

FIGURE 9: The error of flange width and flange height with respect to various wheel positions.

Theoretically, from (14) and (15), we know that the smaller the value of $R$ is, the larger the error $e$ is. So, we chose the largest standard wheelset with $R=385 \mathrm{~mm}$ and $R_{C}=$ $399 \mathrm{~mm}$. Figure 9 shows the error of flange height and flange width induced from misalignment in this case. The distance $d$ varies from $0 \mathrm{~mm}$ to $50 \mathrm{~mm}$ with an interval of $1 \mathrm{~mm}$. From Figure 9, the error of flange height is lower than the error of flange width. So, we focus on the error of flange width here.

In our system, the sampling frequency of all LDS is $1 \mathrm{kHz}$ and the maximum speed of the train in the depot is $36 \mathrm{~km} / \mathrm{h}$. The maximum of sampling step size along the rail $\Delta s=1 \mathrm{~ms} \times$ $10 \mathrm{~m} / \mathrm{s}=10 \mathrm{~mm}$. When we set the threshold $K p$ (as described in Section 2.5) as $20 \mathrm{~mm}$, the total measuring distance along the rail can be $58 \mathrm{~mm}$ so that at least $M=5$ times of efficient scans can be detected. The corresponding errors are less than $0.1 \mathrm{~mm}$ for flange width after taking the average of these 5 efficient scans. Thus, the system can perform detection normally against the misalignment error benefitting from the high sampling frequency.

3.3. Sensor Noise. The LDS cannot be ideally accurate. The measuring accuracy is influenced by temperature, the roughness of the measured surface, and so forth.

In order to obtain the quantitative influence for profile detection, we built a 3D model in SolidWorks tools and extracted ideal sensor output points of standard inner and outer tread profiles. In this model, the standard wheel is located in the position where the center of the wheel is in the laser panel. So, the misalignment phenomenon will not affect tread profile detection. The wheel is in static position so the simulated sensor output points are all from one scan. Moreover, the parameters that need to be calibrated are ideally accurate. To imitate the real situation, Gaussian noise is added to these coordinate values. The mean of noise is zero, and the standard deviation is varied from 0 to $1 \mathrm{~mm}$ with an 


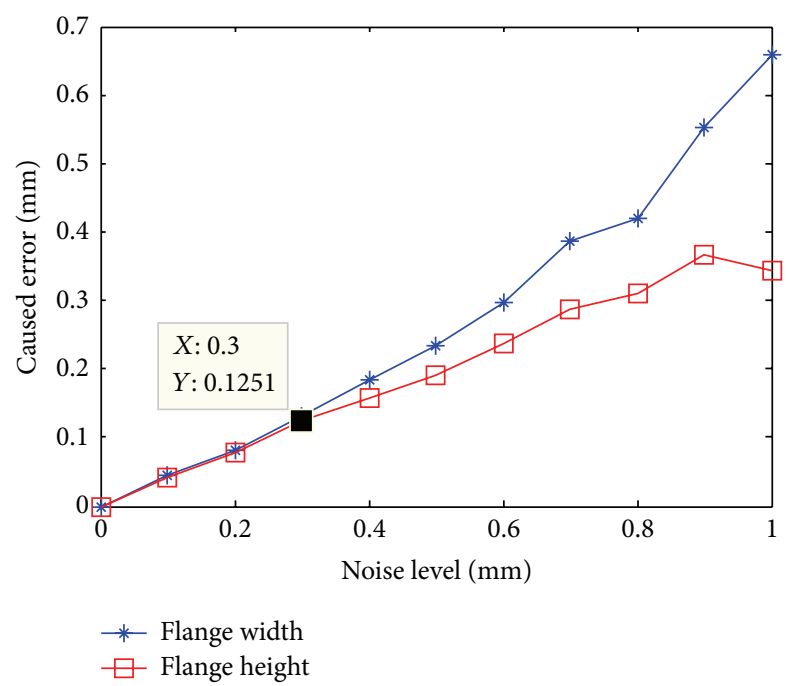

FIGURE 10: The RMS error of flange height and flange width caused by different sensor noise level.

interval of $0.1 \mathrm{~mm}$. For each noise level, 500 experiments are carried out and the RMS error is calculated. The RMS error of flange height and flange width results caused by different noise level is shown in Figure 10. The caused error to flange height and flange width is approximately half of the sensor noise level. This can be explained by the curve line fitting method that has taken more laser points into account and thus has reduced the random noise. Because the flange height is determined by two points, the curve line fitting method has at least reduced the random error into a quarter of the original sensor noise.

The 2D-LDS we chose is LJ-V7300 from @KEYENCE which has a full-scale resolution of $0.1 \% / F . S$ and a temperature drift of $0.01 \%$ F.S $/{ }^{\circ} \mathrm{C}$. The detection range in $y$-axis is $300 \pm 145 \mathrm{~mm}$ and in $x$-axis is $110 \mathrm{~mm}$ to $240 \mathrm{~mm}$, which formed as a trapezoid. The point in $x$-axis is fixed; thus, only sensor noise in $y$-axis needs to be considered with full scale of $290 \mathrm{~mm}$. So, accordingly, the RMS error caused to the profile coordinate noise in $y$-axis, which is denoted by $\delta$, is less than $0.32 \mathrm{~mm}$, which only leads to an error of $0.13 \mathrm{~mm}$ both to flange height and to flange width. Taking dynamics detection effect into account, the final error is reduced by $\delta_{f}=\delta / \sqrt{5}=0.058 \mathrm{~mm}$ with at least $N=5$ times of efficient scans. The error caused by sensor noise can be acceptable.

Regarding the error of wheel diameter, it can be theoretically derived by the theorem of error propagation [25]. The resolution of each sensor is denoted by $\delta_{1}, \delta_{2}$, and $\delta_{3}$. We obtain $\delta_{D}$ by taking differential of (6)-(9) as follows:

$$
\delta_{D}= \pm \sqrt{\left(\delta_{1} \frac{\partial D}{\partial l_{1}}\right)^{2}+\left(\delta_{2} \frac{\partial D}{\partial l_{2}}\right)^{2}+\left(\delta_{3} \frac{\partial D}{\partial l_{3}}\right)^{2}} .
$$

We have chosen two 2D-LDS and one 1D-LDS to detect the wheel diameter and the two 2D-LDS are installed symmetrically. For systematic installation, we have $\delta_{1}\left(\partial D / \partial l_{1}\right)=$ $\delta_{3}\left(\partial D / \partial l_{3}\right)$. Moreover, the analytical function of particle derivative will be too complex to derive. So, we consider a special case where

$$
\begin{aligned}
& {\left[d_{\mathrm{off}}, \alpha_{1}, \alpha_{1}, \alpha_{3}, y_{1}, z_{1}, y_{2}, z_{2}, y_{3}, z_{3}\right]} \\
& \quad=\left[10 \mathrm{~mm}, 45^{\circ}, 90^{\circ}, 135^{\circ},-495 \mathrm{~mm},\right. \\
& \quad-495 \mathrm{~mm}, 0 \mathrm{~mm}, 600 \mathrm{~mm}, 495 \mathrm{~mm},-495 \mathrm{~mm}],
\end{aligned}
$$

where the target wheel diameter is $D=840 \mathrm{~mm}$ and the origin of the wheel is located in the origin of yoz WCF. More calculation details can be found in the Appendix. Finally, we have

$$
\begin{aligned}
& \frac{\partial D}{\partial l_{1}}=-3.4142, \\
& \frac{\partial D}{\partial l_{2}}=4.8284 .
\end{aligned}
$$

The 1D-LDS we chose is LK-G8085 from @KEYENCE which has linearity of $0.05 \% / \mathrm{F}$.S and a temperature drift of $0.01 \%$ F.S $/{ }^{\circ} \mathrm{C}$. So, according to the full scale of $30 \mathrm{~mm}$, the resolution of 1D-LDS $\delta_{2}=0.018 \mathrm{~mm}$. Based upon the finding that the curve line fitting method has at least reduced the random error into a quarter of the original sensor noise, $\delta_{1}=0.075 \mathrm{~mm}$. Finally, $\delta_{D}$ is less than $0.372 \mathrm{~mm}$. Taking dynamics detection effect into account, the final error $\delta_{D_{f}}=$ $\delta_{D} / \sqrt{5}=0.17 \mathrm{~mm}$. The error caused by sensor noise can be acceptable.

3.4. Wheel Inclination Caused by Wheelset S-Shape Running and Differential of Wheel Diameter. In engineering, the wheel will be inclined because of wheelset S-shape running and the differential of wheel diameter. The wheelset S-shape running is one kind of self-induced vibration due to the slope in the wheel trade. When it is S-shape running, the wheel panel will have a certain angle with respect to yoz panel in WCF, denoted by $\theta_{s}$ as shown in Figure 11(a). The differential of wheel diameter in a wheelset is at different wear level in the left and right wheel, mainly induced from different massiveness of wear in the circuit of wheelset turning and unbalanced loading. Similarly, it will bring a certain angle about the wheel panel with respect to the yoz panel in WCF. The angle is denoted by $\theta_{d}$ as shown in Figure 11(b).

For wheel diameter detection, because we only consider the calculation in two dimensions, an error will be generated when we still regard the detected three points in a circle to actually be in an ellipse. Considering the existence of angles $\theta_{s}$ and $\theta_{d}$, we have the equation of ellipse as follows:

$$
\begin{aligned}
& \frac{y^{2}}{\left(R \cdot \cos \theta_{s}\right)^{2}}+\frac{z^{2}}{R^{2}}=1, \\
& \frac{y^{2}}{R^{2}}+\frac{z^{2}}{\left(R \cdot \cos \theta_{d}\right)^{2}}=1 .
\end{aligned}
$$




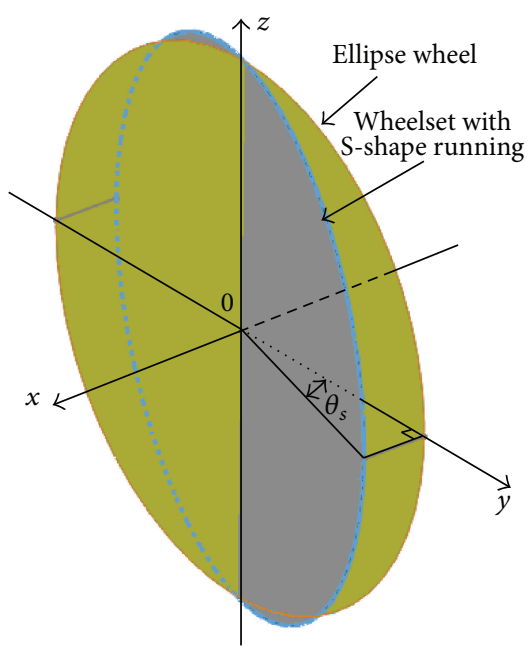

(a)

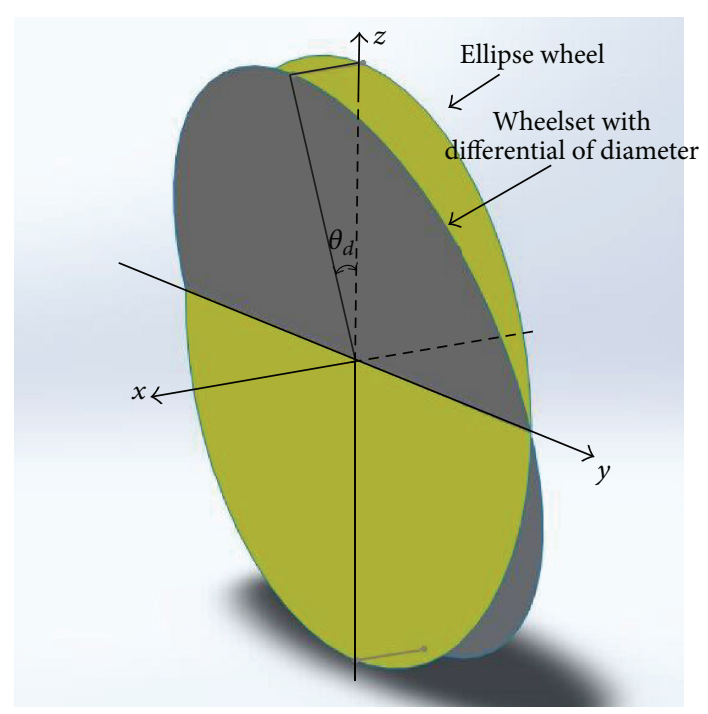

(b)

FIGURE 11: Mathematical illustration. (a) Wheel S-shape running and (b) differential of wheel diameter.

Similar to when we analyze sensor noise, we consider a special case as (17); the origin of the target wheel is located in the origin of $y o z$ WCF. The real three points are

$$
\begin{gathered}
c_{1}:\left(\frac{R \cdot \cos \theta_{s}}{\sqrt{\left(\cos \theta_{s}\right)^{2}+1}},-\frac{R \cdot \cos \theta_{s}}{\sqrt{\left(\cos \theta_{s}\right)^{2}+1}}\right), \\
c_{2}:(0,-R), \\
c_{3}:\left(-\frac{R \cdot \cos \theta_{s}}{\sqrt{\left(\cos \theta_{s}\right)^{2}+1}},-\frac{R \cdot \cos \theta_{s}}{\sqrt{\left(\cos \theta_{s}\right)^{2}+1}}\right), \\
c_{1}:\left(\frac{R \cdot \cos \theta_{d}}{\sqrt{\left(\cos \theta_{d}\right)^{2}+1}},-\frac{R \cdot \cos \theta_{d}}{\sqrt{\left(\cos \theta_{d}\right)^{2}+1}}\right), \\
c_{3}:\left(0,-R \cdot \cos \theta_{d}\right), \\
\left.-\frac{R \cdot \cos \theta_{d}}{\sqrt{\left(\cos \theta_{d}\right)^{2}+1}},-\frac{R \cdot \cos \theta_{d}}{\sqrt{\left(\cos \theta_{d}\right)^{2}+1}}\right) .
\end{gathered}
$$

Theoretically, the larger the radius of the wheel is, the bigger the error is. So, we chose $R=420 \mathrm{~mm}$ and generated three points; then, using (7), we calculated the wheel diameter with error. Subtracting the real diameter, we have the error with respect to angle as shown in Figure 12. The effect of S-shape running caused angle has a relatively higher influence on the wheel diameter calculation.

Based on the experience from Guangzhou Metro Corporation, the differential of diameter in a wheelset should be controlled under $2 \mathrm{~mm}$. Considering the track gauge of $1350 \mathrm{~mm}$, the angle induced from the differential of diameter

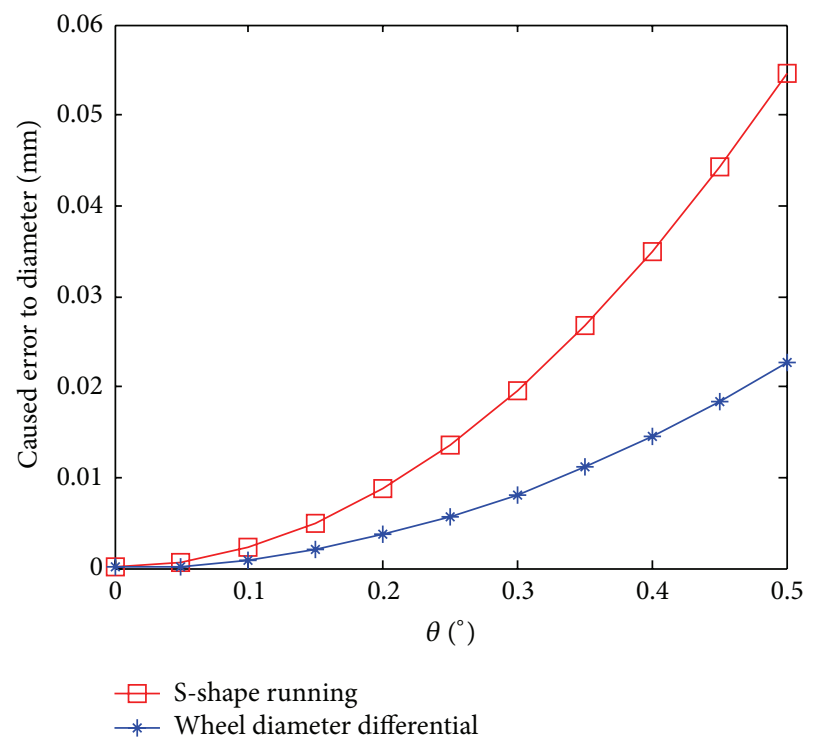

Figure 12: Wheel diameter errors.

in a wheelset is less than $0.001^{\circ}$; thus, the error can be ignored. As for wheel S-shape running, the maximum angle is $0.1^{\circ}$ [26] when the speed of the train is under $36 \mathrm{~km} / \mathrm{h}$ which will cause an error not larger than $0.005 \mathrm{~mm}$.

\section{Experimental Validation}

4.1. System Implementation. The authors previously proposed an online detection system using eight 2D-LDS [19]. The new online detection system is installed in the same storage line of Guangzhou metro vehicle depot as the old system so that comparison can be conducted. In order to save fund, only the left side, namely, half of the system, has been 


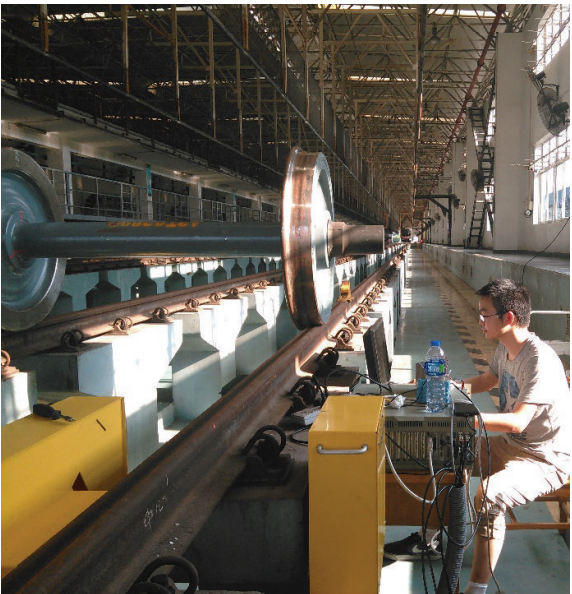

(a)

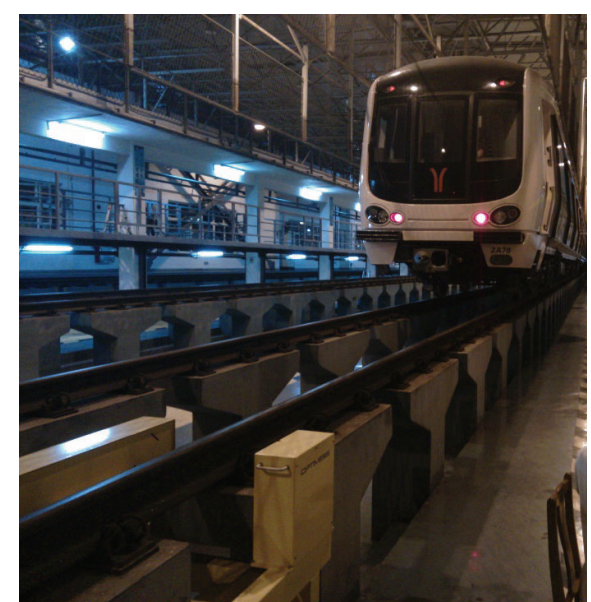

(b)

Figure 13: Field test. (a) Standard wheel test and (b) real train test.

TABLE 1: Standard wheelset detection, the results of repeated measurements $/ \mathrm{mm}$.

\begin{tabular}{lcccccc}
\hline \multirow{2}{*}{$\begin{array}{l}\text { Measurement } \\
\text { times }\end{array}$} & \multicolumn{2}{c}{ Flange height } & \multicolumn{2}{c}{ Flange width } & \multicolumn{2}{c}{ Wheel diameter } \\
& Old & New & Old & New & Old & New \\
\hline 1 & 28.04 & 28.16 & 31.98 & 32.08 & 839.76 & 839.79 \\
2 & 28.11 & 28.21 & 32.06 & 32.01 & 839.96 & 840.15 \\
3 & 27.99 & 28.15 & 32.01 & 32.04 & 840.08 & 839.86 \\
4 & 28.05 & 28.18 & 31.85 & 32.10 & 839.88 & 840.43 \\
5 & 28.08 & 28.16 & 31.93 & 32.02 & 840.01 & 840.04 \\
6 & 28.11 & 28.10 & 32.06 & 32.14 & 839.98 & 840.46 \\
Mean & $\mathbf{2 8 . 0 6}$ & $\mathbf{2 8 . 1 6}$ & $\mathbf{3 1 . 9 8}$ & $\mathbf{3 2 . 0 6}$ & $\mathbf{8 3 9 . 9 5}$ & $\mathbf{8 4 0 . 1 2}$ \\
SD & $\mathbf{0 . 0 4 6}$ & $\mathbf{0 . 0 3 6}$ & $\mathbf{0 . 0 7 8}$ & $\mathbf{0 . 0 5 2}$ & $\mathbf{0 . 1 1 1}$ & $\mathbf{0 . 2 8 1}$ \\
\hline
\end{tabular}

implemented. During the system implementation, threedimensional inclinometer and special rail gauge are used to control the position of the mechanical support. After the system is installed, the calibration described in Section 2 has been conducted to obtain the geometric parameters for tread profile calibration and diameter calculation. As shown in Figure 13, the field test is carried out by a standard wheelset and real train.

4.2. Standard Wheelset. The standard wheelset is a new produced wheelset without any wear and diameter differential. The manufacturing geometric size is as follows: wheel diameter $=840 \mathrm{~mm}$, flange height $=28 \mathrm{~mm}$, and flange width $=$ $32 \mathrm{~mm}$. One can also assume to have lower possibility of Sshape running because of zero external load. The standard wheelset has been placed on the rail and passed through the detection system. This test has been carried out 6 times to verify both the detection and the repeatability of the system. Comparing with the old system, the results of this system are shown in Table 1.

From Table 1, the mean values of the flange height and flange width detected by the old and the new system are very close to each other, which means the system error can be ignored. The standard deviation, which also can be denoted as detection uncertainty, of the new system measurement is slightly smaller than of the old system. That may result from the lower effect from misalignment, as described in Section 3, due to the higher sampling frequency that we used in the new system. Detection uncertainty of not greater than $0.05 \mathrm{~mm}$ in tread profile measurement is acceptable for the engineering requirements. As for wheel diameter detection, the mean values are also close to each other. The standard deviation of the new system measurement is slightly higher than of the old system. This may result from the replacement of $1 D$-LDS that has brought about higher sensor noise to the middle point among three points without curve fitting technique. However, detection uncertainty of less than $0.3 \mathrm{~mm}$ is also acceptable in engineering.

4.3. Real Train Detection Test. Real train test also performs 6 times of repeated detection to statistically evaluate the performance of the system. The train speed is controlled under $36 \mathrm{~km} / \mathrm{h}$. In the train we chose, there are 4 new ground wheelsets in a car of the train. Under the consideration that the ground new wheel is not out of roundness which has an effect on the analysis results, we selected the ground new wheel as our target wheel.

Table 2 shows the mean and standard deviation value of measurement. The biggest differential value of mean flange height appears in \#1 wheel and for mean flange width appears in \#3 wheel. The difference does not exceed $0.15 \mathrm{~mm}$. As for wheel diameter, the biggest differential value $0.16 \mathrm{~mm}$ appears in \#3 wheel. The mean value of six times of repeated detection is consistent with the standard wheelset test. In terms of standard deviation, the value is less than $0.1 \mathrm{~mm}$ for flange width and flange height and $0.3 \mathrm{~mm}$ for wheel diameter. The standard deviation of wheel diameter is relatively higher than in standard wheelset test. On the contrary, the standard deviation of flange width and flange height is relatively lower than in the old system. That is also consistent with standard 
TABLE 2: Real train test, the mean and standard deviation value of detected measurement/mm.

\begin{tabular}{lcccccccccccccc}
\hline \multirow{2}{*}{$\begin{array}{l}\text { Wheel } \\
\text { number }\end{array}$} & \multicolumn{2}{l}{ Mean flange height } & \multicolumn{2}{c}{ SD flange height } & \multicolumn{2}{c}{ Mean flange width } & \multicolumn{2}{c}{ SD flange width } & \multicolumn{3}{c}{$\begin{array}{c}\text { Mean wheel } \\
\text { diameter }\end{array}$} & \multicolumn{2}{c}{ SD wheel diameter } \\
& Old & New & Old & New & Old & New & Old & New & Old & New & Old & New \\
\hline 1 & 28.18 & 28.03 & 0.046 & 0.060 & 29.54 & 29.43 & 0.091 & 0.062 & 800.52 & 801.50 & 0.201 & 0.301 \\
2 & 28.09 & 28.11 & 0.078 & 0.040 & 29.40 & 29.29 & 0.056 & 0.028 & 801.12 & 800.96 & 0.128 & 0.286 \\
3 & 27.97 & 27.91 & 0.076 & 0.033 & 29.92 & 30.06 & 0.075 & 0.056 & 801.87 & 801.66 & 0.090 & 0.179 \\
4 & 28.07 & 28.05 & 0.063 & 0.053 & 29.83 & 29.88 & 0.076 & 0.088 & 801.78 & 802.01 & 0.192 & 0.282 \\
\hline
\end{tabular}

TABLE 3: Real train test, wheel \#2, the result of repeated measure$\mathrm{ment} / \mathrm{mm}$

\begin{tabular}{lcccccc}
\hline \multirow{2}{*}{$\begin{array}{l}\text { Measurement } \\
\text { times }\end{array}$} & \multicolumn{2}{l}{ Flange height } & \multicolumn{2}{c}{ Flange width } & \multicolumn{2}{c}{ Wheel diameter } \\
& Old & New & Old & New & Old & New \\
\hline 1 & 27.98 & 28.15 & 29.42 & 29.31 & 801.07 & 801.40 \\
2 & 28.14 & 28.11 & 29.36 & 29.31 & 801.06 & 800.97 \\
3 & 28.12 & 28.13 & 29.47 & 29.24 & 801.27 & 800.53 \\
4 & 28.10 & 28.12 & 29.31 & 29.29 & 801.06 & 800.87 \\
5 & 28.00 & 28.03 & 29.39 & 29.28 & 800.96 & 800.96 \\
6 & 28.18 & 28.11 & 29.39 & 29.32 & 801.28 & 801.10 \\
Mean & $\mathbf{2 8 . 0 9}$ & $\mathbf{2 8 . 1 1}$ & $\mathbf{2 9 . 4 0}$ & $\mathbf{2 9 . 2 9}$ & $\mathbf{8 0 1 . 1 2}$ & $\mathbf{8 0 0 . 9 6}$ \\
SD & $\mathbf{0 . 0 7 8}$ & $\mathbf{0 . 0 4 0}$ & $\mathbf{0 . 0 5 6}$ & $\mathbf{0 . 0 2 8}$ & $\mathbf{0 . 1 2 8}$ & $\mathbf{0 . 2 8 6}$ \\
\hline
\end{tabular}

TABLE 4: Real train test, wheel \#3, the result of repeated measure$\mathrm{ment} / \mathrm{mm}$.

\begin{tabular}{lcccccc}
\hline \multirow{2}{*}{$\begin{array}{l}\text { Measurement } \\
\text { times }\end{array}$} & \multicolumn{2}{c}{ Flange height } & \multicolumn{2}{c}{ Flange width } & \multicolumn{2}{c}{ Wheel diameter } \\
& Old & New & Old & New & Old & New \\
\hline 1 & 27.90 & 27.90 & 29.78 & 29.99 & 801.95 & 801.59 \\
2 & 28.06 & 27.97 & 29.97 & 30.02 & 801.81 & 801.74 \\
3 & 28.02 & 27.90 & 29.91 & 30.05 & 801.96 & 801.48 \\
4 & 27.99 & 27.90 & 29.98 & 30.09 & 801.78 & 801.48 \\
5 & 28.00 & 27.87 & 29.94 & 30.15 & 801.95 & 801.94 \\
6 & 27.86 & 27.90 & 29.96 & 30.06 & 801.78 & 801.74 \\
Mean & $\mathbf{2 7 . 9 7}$ & $\mathbf{2 7 . 9 1}$ & $\mathbf{2 9 . 9 2}$ & $\mathbf{3 0 . 0 6}$ & $\mathbf{8 0 1 . 8 7}$ & $\mathbf{8 0 1 . 6 6}$ \\
SD & $\mathbf{0 . 0 7 6}$ & $\mathbf{0 . 0 3 3}$ & $\mathbf{0 . 0 7 5}$ & $\mathbf{0 . 0 5 6}$ & $\mathbf{0 . 0 9 0}$ & $\mathbf{0 . 1 7 9}$ \\
\hline
\end{tabular}

wheelset test. The standard deviation of wheel diameter in real train test is supposed to be higher than in the standard wheelset test because of several assumptions. One factor is the higher possibility of S-shape running because of heavy axial load. On the other hand, the wheelset that is in service is also more polluted with rust than standard wheelset, causing more detection uncertainty. However, the standard deviation from real train test also does not exceed $0.3 \mathrm{~mm}$, which is consistent with standard wheelset test. This may result from the lower train speed during the test which leads to lower possibility of S-shape running. Meanwhile, the rusty wheel contour is also not in a massive stage. Tables 3 and 4 show the result of repeated measurement for wheels numbers 2 and 3 , respectively. In each detection, the results remain the same and no gross error appears.
Overall, detection uncertainties for tread profile and wheel diameter are less than $0.1 \mathrm{~mm}$ and $0.3 \mathrm{~mm}$, respectively. The results show that the detection system has a high accuracy, which can meet the requirements of maintenance operation.

\section{Conclusion}

This paper, based on LDS, proposed a novel on-track detection system of the wheel size using only six 2D-LDS and two 1D-LDS. Errors induced by wheel-rail vibration, sensor noise, misalignment, S-shape running, and wheelset differential are also analyzed. After the system is implemented, real data experiments including standard wheel test and real train detection test were performed. It turns out that the detection uncertainty of flange width and height is $0.1 \mathrm{~mm}$ and wheel diameter $0.3 \mathrm{~mm}$, which can meet the requirements of maintenance. This system can be further used for different types of railway transportation, which provides a new solution for the wheel size detection technology.

\section{Appendix}

We consider a special case where

$$
\begin{aligned}
& {\left[d_{\text {off }}, \alpha_{1}, \alpha_{1}, \alpha_{3}, y_{1}, z_{1}, y_{2}, z_{2}, y_{3}, z_{3}\right]} \\
& \quad=\left[10 \mathrm{~mm}, 45^{\circ}, 90^{\circ}, 135^{\circ},-495 \mathrm{~mm},\right. \\
& \quad-495 \mathrm{~mm}, 0 \mathrm{~mm}, 600 \mathrm{~mm}, 495 \mathrm{~mm},-495 \mathrm{~mm}] .
\end{aligned}
$$

To provide more benefits, the target wheel diameter is $D=$ $840 \mathrm{~mm}$ and the origin of the wheel is located in the origin of $y o z$ WCF, as shown in Figure 14. In this special case, the relevant geometric values are $c_{1}(-198.02 \mathrm{~mm},-198.02 \mathrm{~mm})$, $c_{2}(0 \mathrm{~mm}, 420 \mathrm{~mm}), c_{3}(198.02 \mathrm{~mm},-198.02 \mathrm{~mm}), l_{1}=280 \mathrm{~mm}$, $l_{2}=180 \mathrm{~mm}$, and $l_{3}=280 \mathrm{~mm}$.

According to (8) and (9), we get the particle derivative as follows:

$$
\begin{aligned}
& \frac{\partial D}{\partial l_{1}}=\frac{\partial D}{\partial y_{0}} \frac{\partial y_{0}}{\partial l_{1}}+\frac{\partial D}{\partial z_{0}} \frac{\partial z_{0}}{\partial l_{1}}, \\
& \frac{\partial D}{\partial l_{2}}=\frac{\partial D}{\partial y_{0}} \frac{\partial y_{0}}{\partial l_{2}}+\frac{\partial D}{\partial z_{0}} \frac{\partial z_{0}}{\partial l_{2}}+\frac{\partial D}{\partial z_{\mathcal{c}_{2}}} \frac{\partial z_{\mathcal{G}_{2}}}{\partial l_{2}} .
\end{aligned}
$$




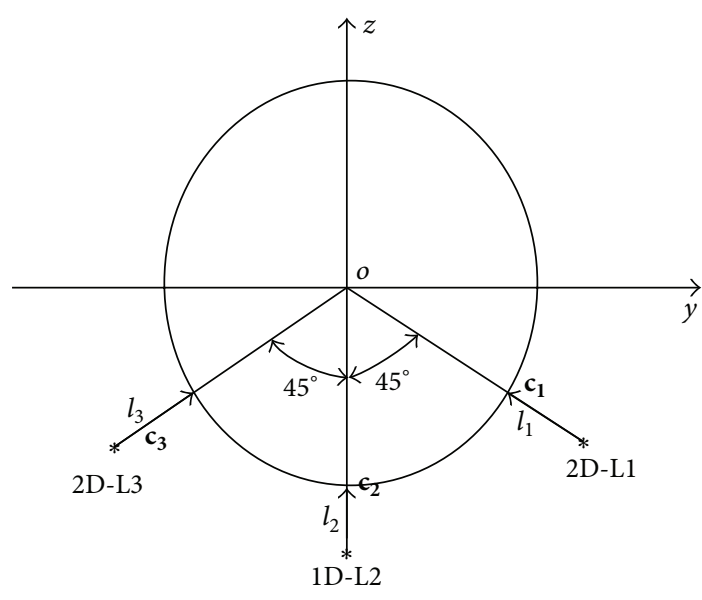

Figure 14: A special case.

Taking the derivative of diameter $D$ with respect to $y_{0}, z_{0}$, and $z_{c_{1}}$ according to $(8)$ and substituting $\left(y_{0}, z_{0}\right)=(0,0)$ and $z_{\mathcal{c}_{2}}=180 \mathrm{~mm}$, we have

$$
\begin{aligned}
& \frac{\partial D}{\partial y_{0}}=\frac{2 y_{0}}{\sqrt{\left(y_{0}\right)^{2}+\left(z_{0}-z_{\mathcal{c}_{2}}\right)^{2}}}=\frac{1}{180 \mathrm{~mm}}, \\
& \frac{\partial D}{\partial z_{0}}=\frac{2\left(z_{0}-z_{\mathcal{c}_{2}}\right)}{\sqrt{\left(y_{0}\right)^{2}+\left(z_{0}-z_{\mathcal{C}_{2}}\right)^{2}}}=\frac{1}{180 \mathrm{~mm}}, \\
& \frac{\partial D}{\partial z_{\mathcal{C}_{2}}}=\frac{-2\left(z_{0}-z_{\left.\mathcal{c}_{2}\right)}\right.}{\sqrt{\left(y_{0}\right)^{2}+\left(z_{0}-z_{\mathcal{c}_{2}}\right)^{2}}}=2 .
\end{aligned}
$$

Furthermore, based upon (7), we get

$$
\begin{aligned}
& \frac{\partial y_{0}}{\partial l_{1}}=\frac{\partial y_{0}}{\partial y_{c_{1}}} \frac{\partial y_{c_{1}}}{\partial l_{1}}+\frac{\partial y_{0}}{\partial z_{c_{1}}} \frac{\partial z_{c_{1}}}{\partial l_{1}}, \\
& \frac{\partial z_{0}}{\partial l_{1}}=\frac{\partial z_{0}}{\partial y_{c_{1}}} \frac{\partial y_{c_{1}}}{\partial l_{1}}+\frac{\partial z_{0}}{\partial z_{c_{1}}} \frac{\partial z_{c_{1}}}{\partial l_{1}}, \\
& \frac{\partial y_{0}}{\partial l_{2}}=\frac{\partial y_{0}}{\partial z_{\mathcal{c}_{2}}} \frac{\partial z_{\mathcal{c}_{2}}}{\partial l_{2}}, \\
& \frac{\partial z_{0}}{\partial l_{2}}=\frac{\partial z_{0}}{\partial z_{\mathcal{c}_{2}}} \frac{\partial z_{\mathcal{c}_{2}}}{\partial l_{2}}, \\
& \frac{\partial y_{0}}{\partial l_{3}}=\frac{\partial y_{0}}{\partial y_{\mathcal{c}_{3}}} \frac{\partial y_{\mathcal{c}_{3}}}{\partial l_{3}}+\frac{\partial y_{0}}{\partial z_{\mathcal{c}_{3}}} \frac{\partial z_{\mathcal{c}_{3}}}{\partial l_{3}}, \\
& \frac{\partial z_{0}}{\partial l_{3}}=\frac{\partial z_{0}}{\partial y_{c_{3}}} \frac{\partial y_{\mathcal{c}_{3}}}{\partial l_{3}}+\frac{\partial z_{0}}{\partial z_{\mathcal{c}_{3}}} \frac{\partial z_{\mathcal{c}_{3}}}{\partial l_{3}} .
\end{aligned}
$$

When calculating particle derivative of $\left(y_{0}, z_{0}\right)$ with respect to three points $c_{1}, c_{2}$, and $c_{3}$ in WCF, we assume that all parameters are with the geometric values in this special case.
Then, we substitute the ideal geometric values of this variable, and we obtain

$$
\begin{gathered}
\frac{\partial y_{0}}{\partial y_{c_{1}}}=0.5 \\
\frac{\partial y_{0}}{\partial z_{c_{1}}}=0.5
\end{gathered}
$$$$
\frac{\partial z_{0}}{\partial y_{c_{1}}}=-1.2071 \text {, }
$$$$
\frac{\partial z_{0}}{\partial z_{c_{1}}}=-1.2071,
$$$$
\frac{\partial y_{0}}{\partial z_{c_{2}}}=0,
$$$$
\frac{\partial z_{0}}{\partial z_{c_{2}}}=3.4142,
$$$$
\frac{\partial y_{0}}{\partial y_{c_{3}}}=0.5 \text {, }
$$$$
\frac{\partial y_{0}}{\partial z_{c_{3}}}=-0.5 \text {, }
$$

$\frac{\partial z_{0}}{\partial y_{c_{3}}}=1.2071$,

$\frac{\partial z_{0}}{\partial z_{c_{3}}}=-1.2071$,

$\frac{\partial y_{c_{1}}}{\partial l_{1}}=\frac{1}{\sqrt{2}}$,

$\frac{\partial z_{c_{1}}}{\partial l_{1}}=\frac{1}{\sqrt{2}}$,

$\frac{\partial z_{c_{2}}}{\partial l_{2}}=-1$,

$\frac{\partial y_{c_{3}}}{\partial l_{3}}=-\frac{1}{\sqrt{2}}$,

$$
\frac{\partial z_{\mathcal{c}_{3}}}{\partial l_{3}}=\frac{1}{\sqrt{2}} \text {. }
$$

Finally, substituting (A.5) into (A.4) and then substituting (A.4) and (A.3) into (A.2), we have

$$
\begin{aligned}
& \frac{\partial D}{\partial l_{1}}=-3.4142, \\
& \frac{\partial D}{\partial l_{2}}=4.8284 .
\end{aligned}
$$

\section{Competing Interests}

The authors declare that they have no competing interests. 


\section{Acknowledgments}

This research was carried out under the National Key Research and Development Plan of China (2016YFB1200402), the Science and Technology Program of Guangzhou (201508010010), and the Fundamental Research Funds for the Central Universities (AE89454). The fund is greatly acknowledged. Special thanks are due to Mr. Jie Jiang for his help in 3D design in SolidWorks.

\section{References}

[1] Y. Chen, Z. Xing, J. Li, and Y. Qin, "The analysis of wheel-rail vibration signal based on frequency slice wavelet transform," in Proceedings of the 17th IEEE International Conference on Intelligent Transportation Systems (ITSC '14), pp. 1312-1316, Qingdao, China, October 2014.

[2] R. Pohl, A. Erhard, H.-J. Montag, H.-M. Thomas, and H. Wüstenberg, "NDT techniques for railroad wheel and gauge corner inspection," NDT \& E International, vol. 37, no. 2, pp. 89-94, 2004.

[3] The International Union of Railways, UIC 510-2 Code. Trailing Stock: Wheels and Wheelsets. Conditions Concerning the Use of Wheels of Various Diameters, The International Union of Railways, Paris, France, 2004.

[4] Z. Zhang, C. Lu, F. Zhang, Y. Ren, K. Yang, and Z. Su, "A novel method for non-contact measuring diameter parameters of wheelset based on wavelet analysis," Optik, vol. 123, no. 5, pp. 433-438, 2012.

[5] Web-1, 2016, https://www.greenwood.dk/miniprofwheel.php.

[6] S. O. Medianu, G. A. Rimbu, D. Lipcinski, I. Popovici, and D. Strambeanu, "System for diagnosis of rolling profiles of the railway vehicles," Mechanical Systems and Signal Processing, vol. 48, no. 1-2, pp. 153-161, 2014.

[7] Web-2, http://www.mermecgroup.com/inspection-technology/ train-monitoring/87/1/wheel-profile-and-diameter.php.

[8] Web-3, http://iem.net/freight-rail-40478?id=150.

[9] Web-4, 2016, http://www.kldlabs.com/index.php?s=wheel+profile+measurement.

[10] X. Chen, J. Sun, Z. Liu, and G. Zhang, "Dynamic tread wear measurement method for train wheels against vibrations," Applied Optics, vol. 54, no. 17, pp. 5270-5280, 2015.

[11] Z. Gong, J. Sun, and G. Zhang, "Dynamic structured-light measurement for wheel diameter based on the cycloid constraint," Applied Optics, vol. 55, no. 1, pp. 198-207, 2016.

[12] Z. F. Mian, J. C. Mullaney, R. MacAllister, and T. J. Schneider, “Optical wheel evaluation," U.S. Patent No. 7,564,569, 2009.

[13] Y. Gao, S. Shao, and Q. Feng, "A new method for dynamically measuring diameters of train wheels using line structured light visual sensor," in Proceedings of the International Symposium on Photonics and Optoelectronics (SOPO '12), pp. 1-4, IEEE, Shanghai, China, May 2012.

[14] Z.-F. Zhang, Z. Gao, Y.-Y. Liu et al., "Computer vision based method and system for online measurement of geometric parameters of train wheel sets," Sensors, vol. 12, no. 1, pp. 334346, 2012.

[15] A. N. Baibakov, K. I. Kuchinskii, V. I. Paterikin, S. V. Plotnikov, and V. V. Sotnikov, "Experience in developing and using automated laser diagnostic equipment for the contactless monitoring of the parameters of freight car wheels," Measurement Techniques, vol. 53, no. 4, pp. 444-448, 2010.
[16] Yu. N. Dubnishchev, P. Y. Belousov, O. P. Belousova, and V. V. Sotnikov, "Optical control of the radius of a wheel rolling on a rail," Optoelectronics, Instrumentation and Data Processing, vol. 48, no. 1, pp. 75-80, 2012.

[17] Y. Gao, Q. Feng, and J. Cui, "A simple method for dynamically measuring the diameters of train wheels using a onedimensional laser displacement transducer," Optics and Lasers in Engineering, vol. 53, pp. 158-163, 2014.

[18] K. Wu and J. Chen, "Dynamic measurement for wheel diameter of train based on high-speed CCD and laser displacement sensors," Sensor Letters, vol. 9, no. 5, pp. 2099-2103, 2011.

[19] Z. Zhang, Z. Su, Y. Su, and Z. Gao, "Denoising of sensor signals for the flange thickness measurement based on wavelet analysis," Optik-International Journal for Light and Electron Optics, vol. 122, no. 8, pp. 681-686, 2011.

[20] Z. Xing, Y. Chen, X. Wang, Y. Qin, and S. Chen, "Online detection system for wheel-set size of rail vehicle based on 2D laser displacement sensors," Optik, vol. 127, no. 4, pp. 1695-1702, 2016.

[21] CN-TB, “Tread profile for locomotive and car," 2003.

[22] A. Ravindran, K. M. Ragsdell, and G. V. Reklaitis, Engineering Optimization: Methods and Applications, John Wiley \& Sons, New York, NY, USA, 2nd edition, 2006.

[23] T. J. Ko, J. W. Park, H. S. Kim, and S. H. Kim, "On-machine measurement using a noncontact sensor based on a CAD model," The International Journal of Advanced Manufacturing Technology, vol. 32, no. 7-8, pp. 739-746, 2007.

[24] C. Zou, Y. Wang, P. Wang, and J. Guo, "Measurement of ground and nearby building vibration and noise induced by trains in a metro depot," Science of the Total Environment, vol. 536, pp. 761-773, 2015.

[25] A. J. Wheeler and A. R. Ganji, Introduction to Engineering Experimentation, Prentice Hall, Upper Saddle River, NJ, USA, 3rd edition, 2010.

[26] A. Qin, M. Su, and Y. Yao, "Influence of hunting wave to lateral vibration of deck steel plate bridges," Journal of Shijiazhuang Railway Institute, vol. 20, no. 1, pp. 56-60, 2007. 


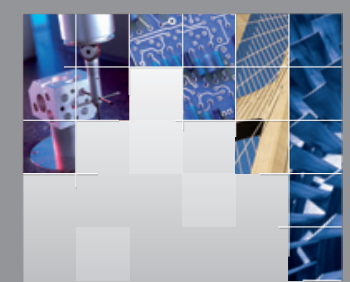

\section{Enfincering}
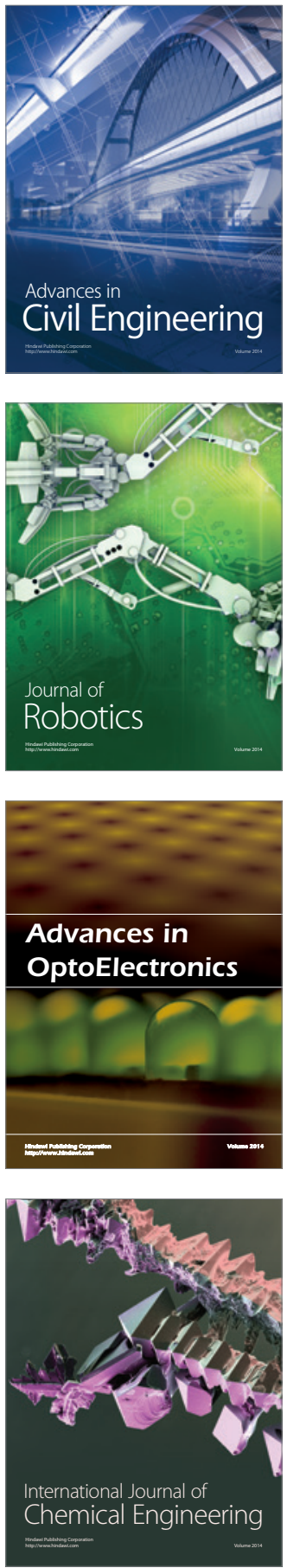

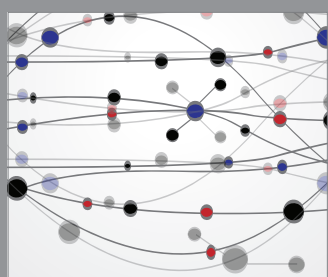

The Scientific World Journal

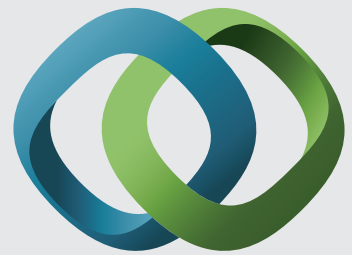

\section{Hindawi}

Submit your manuscripts at

http://www.hindawi.com
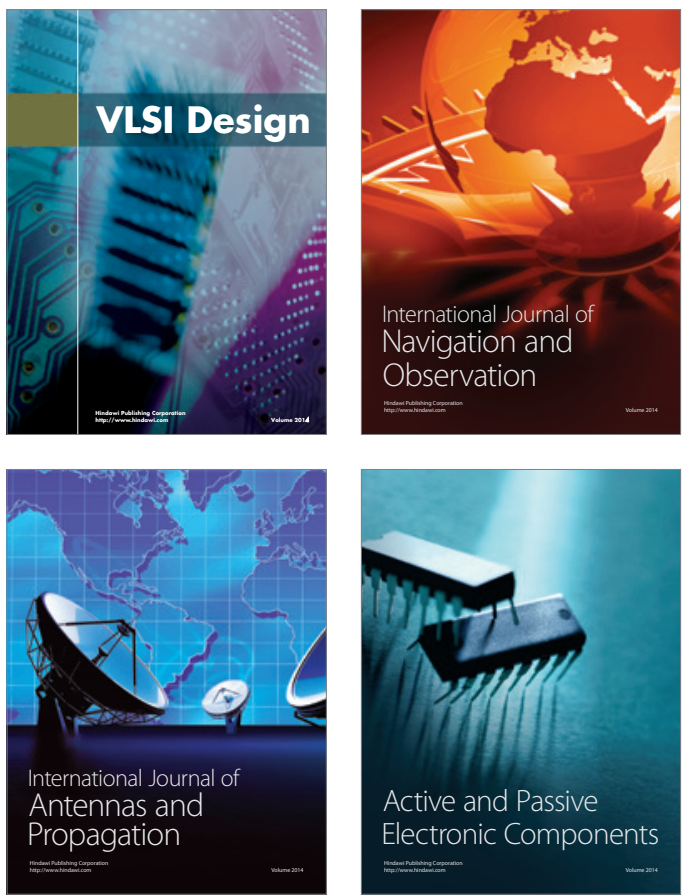


International Journal of

Distributed

Sensor Networks

Journal of

Control Science

and Engineering
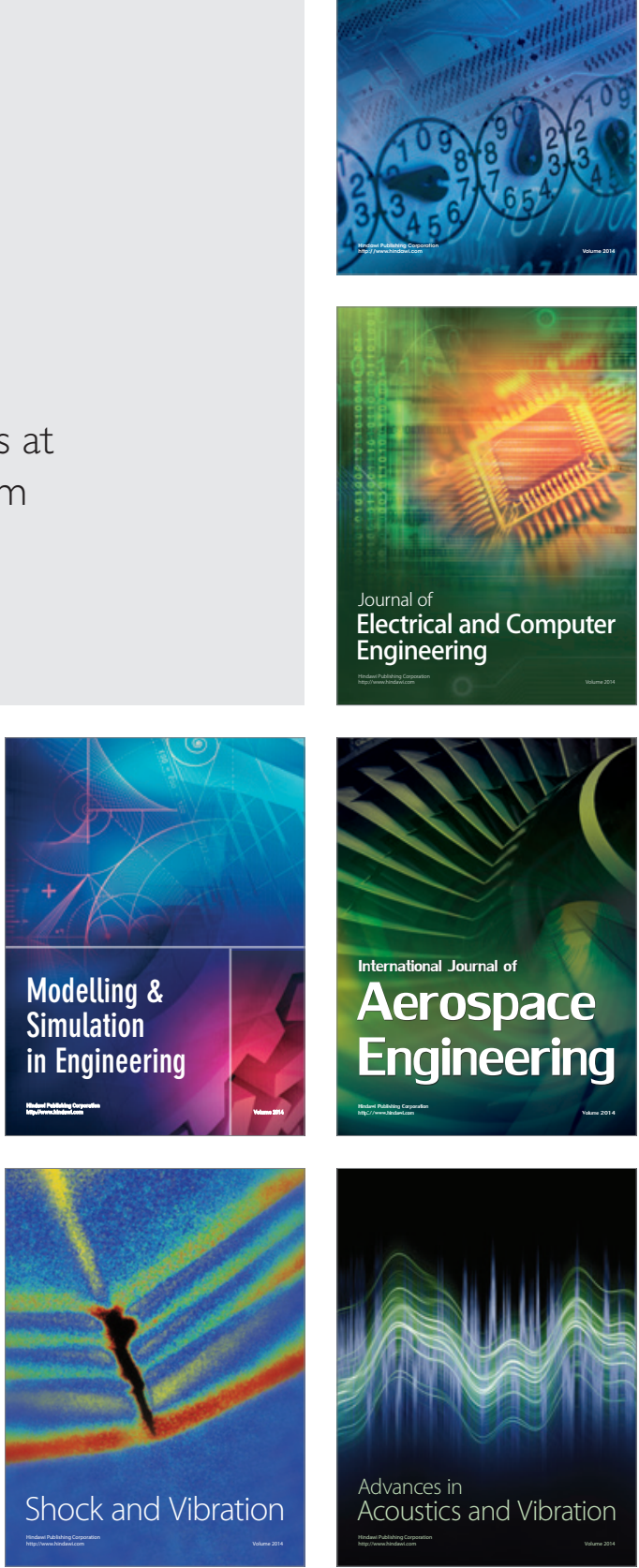